\title{
On some common features of drop impact on liquid surfaces
}

\author{
Alexander I. Fedorchenko ${ }^{\text {a) }}$ and An-Bang Wang ${ }^{\text {b) }}$ \\ Institute of Applied Mechanics, National Taiwan University, Taipei 106, Taiwan, Republic of China
}

(Received 10 December 2002; accepted 12 January 2004; published online 5 April 2004)

\begin{abstract}
The impact of a drop on liquid surfaces is studied experimentally and theoretically in the region of the fully developed splashing. In order to reveal the influence of viscosity and target liquid depth on the resulting flow patterns, the experiments were carried out with water and $70 \%$ glycerol-water solution, and for different target liquid depths. Based on the experimental observations, a dynamic model of the central jet formation at the cavity collapse is developed. This model predicts an emergence of a liquid flow up into the central jet and simultaneously a small flow velocity downward and allows us to evaluate the velocities of these two flows. A theoretical model for the cavity submergence is presented. This model gives the constant velocity of the cavity submergence which is half the initial drop impact velocity. Analytical solution for the gravity-capillary cavity collapse has been derived and provides a good fit to the experimental results. Theoretical analysis and experiments have shown that the maximum cavity radius and the cavity collapse time depend on both the Froude number and the dimensionless capillary length. () 2004 American Institute of Physics. [DOI: 10.1063/1.1652061]
\end{abstract}

\section{INTRODUCTION}

In spite of more than a century of experimental study of the drop impact on liquid surfaces, starting in the last century by Worthington, ${ }^{1}$ we are still far from a detailed understanding of the physical mechanisms causing the observed phenomena.

For high-speed impact of the large water drop on water liquid, Engel ${ }^{2,3}$ carried out a quantitative investigation and derived an equation for the cavity depth on the basis of the experimental evidences. In deriving this equation, an energy consideration based on an empirical approximation for potential energy of surface in the limit of high-speed impact was used. The approximation is not valid for low and moderate drop impact velocities.

For low and moderate drop impact velocities, $\operatorname{Rein}^{4}$ offered a qualitative classification of different regimes of drop impacts, such as splashing, coalescing, floating, and bouncing. Based on experiments with water drops impacting on water target liquid, $\operatorname{Rein}^{5}$ gave a qualitative classification of the different types of fluid flow in the transitional regime between coalescence and splashing. He noticed that nondimensional number like capillary number is needed to describe the transition processes and drop impacts in the region of regular entrainment are always connected with the ejection of a thin high-rising jet out of the collapsing cavity.

Pumphrey and Elmore ${ }^{6}$ evaluated the maximum size of the cavity by equating the stored potential energy of cavity to the kinetic energy of the impacting drop. The obtained dimensional expression for the maximum cavity size $R_{m}$ is a

\footnotetext{
${ }^{a)}$ Permanent address: S. S. Kutateladze Institute of Thermophysics, Siberian Branch, Russian Academy of Sciences, 1 Acad. Lavrentyev Ave., 630090, Novosibirsk, Russia.

b) Author to whom correspondence should be addressed; electronic mail: abwang@ spring.iam.ntu.edu.tw
}

power function of the drop impact velocity $U$ and drop diameter $D$. In their experiments they observed a capillary wave which travels down along the cavity walls. Recently, Morton $\mathrm{et} \mathrm{al}^{7}$ showed that the existence of capillary waves is a necessary condition for bubble entrainment.

Shin and $\mathrm{McMahon}^{8}$ revealed that the cavity collapse of drop impact on shallow liquid layers is similar to a cavity collapse leading to the entrainment of a bubble in deep liquids, if the depth of the target liquid layer is such that the cavity at its maximum size just touches the bottom. They proposed that the formation of the cylindrical stem at the bottom of the cavity is necessary for the ejection of thin jets in shallow liquid layers.

Prosperetti and $\mathrm{Oguz}^{9}$ offered a dimensionless expression for the maximum cavity size in the following form:

$$
R_{m}^{*}=R_{m} / D=(\mathrm{Fr} / 3)^{1 / 4},
$$

where $\mathrm{Fr}=U^{2} /(g D)$ is the Froude number; $D, U$, and $g$ are the drop diameter, impact velocity, and acceleration of gravity, respectively. Liow ${ }^{10}$ found that the cavity sizes in Pumphrey and Elmore ${ }^{6}$ are significantly larger than those in any other published results and one-third of the results were on or above the theoretical line, which indicated that the cavity's potential energy was either equal or larger than that supplied by the drop kinetic energy. Liow ${ }^{10}$ noticed that Eq. (1) overpredicts essentially the maximum cavity size. To correct it, he applied the same scaling $\mathrm{Fr}^{1 / 4}$ with a multiple constant by using a least-square fit to experimental data and gave

$$
R_{m}^{*}=0.727(\mathrm{Fr} / 3)^{1 / 4}
$$

From Eq. (2), Liow ${ }^{10}$ reasoned that only $28 \%$ of the impacting drop kinetic energy was converted to the cavity potential energy. It is worthy to note that the experiments in Liow ${ }^{10}$ were conducted with water drop diameter of $2.055 \mathrm{~mm}$ $\pm 10 \%$, and the corresponding dimensionless capillary 
TABLE I. Property of fluids used in experiments.

\begin{tabular}{lccc}
\hline \hline \multicolumn{1}{c}{$t=2{ }^{\circ} \mathrm{C}$} & $\begin{array}{c}\text { Specific gravity, } \\
\mathrm{kg} / \mathrm{m}^{3}\end{array}$ & Viscosity, $\mathrm{N} \mathrm{s} / \mathrm{m}^{2}$ & $\begin{array}{c}\text { Surface tension, } \\
\mathrm{N} / \mathrm{m}\end{array}$ \\
\hline Distilled water & 1000 & 0.001 & 0.0728 \\
$70 \%$ gl sol & 1200 & 0.0218 & 0.0641 \\
\hline \hline
\end{tabular}

length $l_{c}^{*}=[2 \sigma /(\rho g)]^{1 / 2} / D$ was about $1.86 \pm 10 \%$. Here $\sigma$ and $\rho$ are surface tension and density of the drop. According to Landau and Lifshitz, ${ }^{11}$ for $l_{c}^{*}>1$, the capillary phenomena begin to play an important role and have to be taken into account. Since Eq. (2) does not depend on $l_{c}^{*}$, the validity of Eq. (2) in the range of $l_{c}^{*}>1$ is thus questionable.

The present work, first of all, attempts to reveal the influence of the capillary forces, which is commonly omitted in the literature, on the common features of drop impact on liquid surfaces, e.g., maximum cavity size, and the cavity collapse dynamic. We provide new experimental observations in the region of the fully developed splashing. Based on the experimental observations, the influence of viscosity and the target liquid depth on secondary drops and central jet formation is also discussed.

\section{EXPERIMENTAL DESCRIPTIONS}

Experiments were carried out for different drop/target liquid combinations: water/water, $70 \%$ glycerol-water solution (hereinafter $\mathrm{gl} \mathrm{sol}$ )/water and $\mathrm{gl} \mathrm{sol} / \mathrm{gl}$ sol. The dimensionless target liquid depth $\left(H^{*}=H / D\right)$ varies from 0.5 to 6 . The length is scaled by the drop diameter, $D$, and time by $D / U$, where $U$ is the drop velocity before impact. The fluid properties, such as the density $(\rho)$, the viscosity $(\mu)$, and the surface tension $(\sigma)$ are given in Table I. The experimental study of the drop impacts on very thin liquid films with $H^{*}=0.05$ was reported previously. ${ }^{12}$

The target liquid was contained in a square glass tank, $400 \mathrm{~mm}$ on each side. All runs were carried out by keeping the drop diameter constant and increasing the impact velocity. To keep the drop size stabilized, a constant head vessel filled with working fluid was connected to the drop generator. Single drops were formed through a well-polished glass nozzle and letting them detach under their own weight. The drop diameters were $3.64 \mathrm{~mm}$ for $\mathrm{gl} \mathrm{sol}$ and $3.12 \mathrm{~mm}$ for water.

The photographs were taken with the high-speed CCD camera and Kodak Motion corder analyzer. We normally used the frame rate 1000 frame per second, the shutter speed was $1 / 5000$ second and the resolution of the frame was 256 $\times 240$ pixels. A 1000-watt halogen lamp was used for experimental illumination.

\section{QUALITATIVE ANALYSIS OF SPLASHING PHENOMENA OF DROP IMPACT}

For a water drop impacting a water target liquid in the fully developed splashing region, that is, impact with moderately high energy, the following sequence of events commonly takes place as shown in Figs. 1(a)-1(j). After the drop has collided with the free surface of the target liquid, a flared

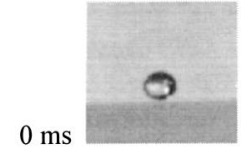

(a)

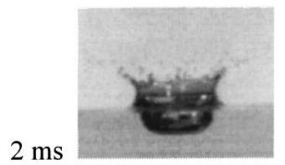

(b)

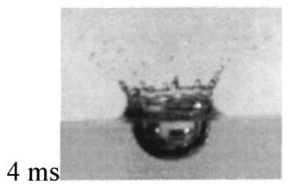

(c)

(d)

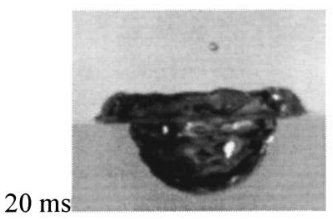

(e)

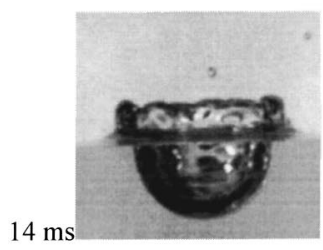

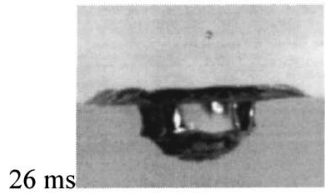

(f)

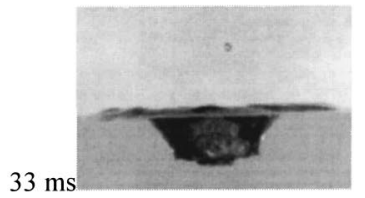

(g)

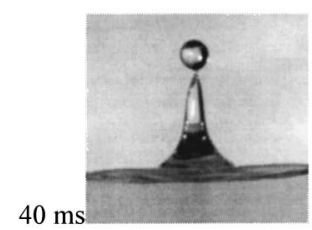

(h)

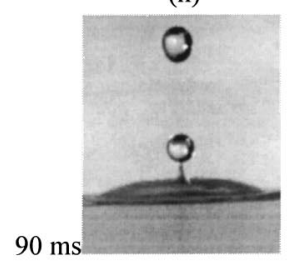

(i)

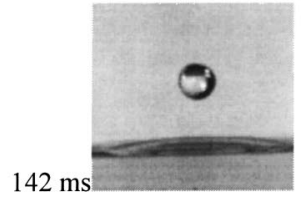

(j)
FIG. 1. Sequence of a water drop impacting on a water target liquid. The impact conditions are $D=3.12 \mathrm{~mm}, U=3.47 \mathrm{~m} / \mathrm{s}, H=15 \mathrm{~mm}, F r=393$, and $l_{c}^{*}=1.22$.

film of liquid is thrown upward and outward from the periphery of the colliding region [see Fig. 1(b)]. The flared film of liquid contains primarily the impacting drop, and part of the target liquid as well. The rim is formed at the top of the film. The capillary wave train has been observed in Figs. 1(b) and 1(c) [see also Figs. 2(a) and 2(b)] and propagates along the film. Below the level of the original free liquid surface a cavity begins to develop. The visible part on the surface of the cavity in these figures is the drop-air interface.

Small liquid jets shoot out from the upper rim of the film and giving the appearance of a crown. According to Rein, ${ }^{5}$ the appearance of a crown is characteristic of splashing in its fully developed form. These jets break down into some small liquid fragments or the so-called secondary droplets [see Figs. 1(b) and 1(c)]. The cavity continues to enlarge and soon takes the shape of a hemisphere [see Fig. 1(c)]. The crown wall begins to subside and becomes thicker [Fig. 1(d)]. The cavity stops growing downwards and starts to collapse [Fig. 1(e)]. At this moment the capillary waves begin to spread over the cavity and thus distort its shape. The cavity 


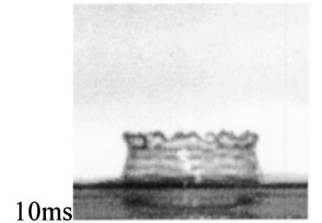

(a)

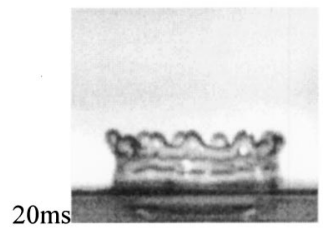

(b)

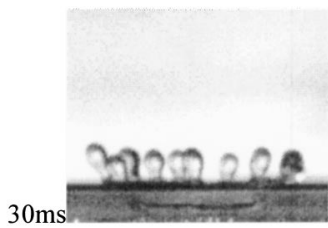

(c)

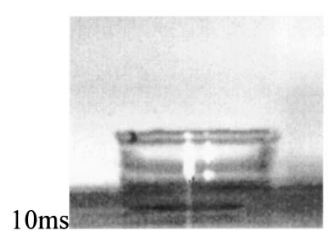

(d)

(e)

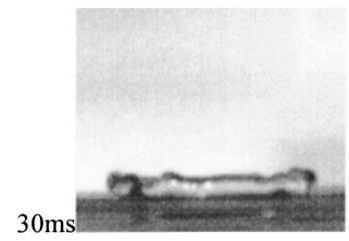

(f)

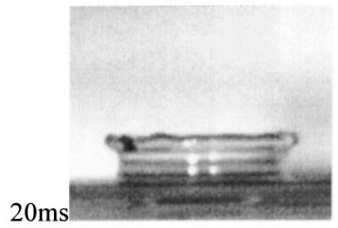

FIG. 2. Gl sol drop impacts on shallow liquid layers of water [frames (a)(c)] and gl sol [frames (d)-(f)]. The impact conditions are $\mathrm{We}=381, l_{c}^{*}$ $=0.91$, and $H^{*}=0.5$.

takes the shape of two- or three-floor "amphitheater" with a very sharp edge between the adjacent floors [see Fig. 1(f)]. The cavity starts to close in from the bottom so that the depth of the cavity quickly decreases [see Figs. $1(\mathrm{f})$ and $1(\mathrm{~g})$ ]. In the meanwhile, the width of the crater does not change significantly, that is, the liquid is accelerated mostly upward within this period. The cavity assumes a nearly cylindrical shape at the instant before the central jet emerges [see Fig. $1(\mathrm{~g})]$. The cavity collapse of this shape results in a thick slow jet rising out of the cavity. After the central jet reaches its greatest height as shown in Fig. 1(h), a blob has formed at the tip of the central jet as shown in Fig. 1(h). The jet pinches off and forms two secondary drops [Fig. 1(i)]. The size of the first secondary drop is close to the size of the original drop [cf. Figs. 1(a) and 1(k)].

To compare the drop impact on low and high viscous target liquids with the same thickness $H^{*}=0.5$, Fig. 2 shows the impact of gl sol drop on water (left column) and gl sol (right column) target liquids, respectively. The impact conditions were similar in both cases. It becomes evident from Fig. 2 that the viscosity has strong effect on behavior of the capillary train along the wall of a cylindrical wave that rises at the cavity periphery and the process of secondary droplets formation. The source of the capillary waves is the enlarging rim at the edge of this cylindrical wave. As shown in Figs. 2(a) and 2(b) for the case of impact on water, the capillary wave propagates downwards along the crown wall and the perturbations of the rim grow quickly, causing the rim "explosion" [see Fig. 2(c)]. The situation changes dramatically for drop impact on gl sol target liquid: capillary waves are damped due to high viscosity of the liquid and the rim distorts weakly [see Figs. 2(d)-2(f)]. It is worthy to note that in both cases cylindrical waves stay perpendicular to the sur-

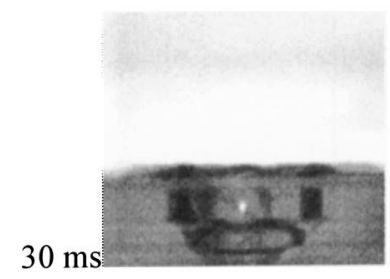

1

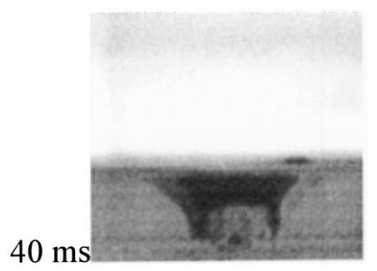

2

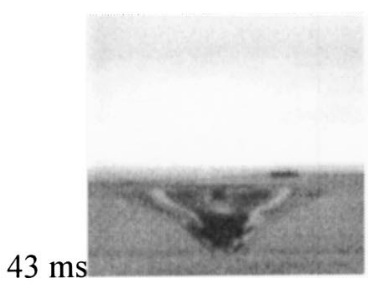

3

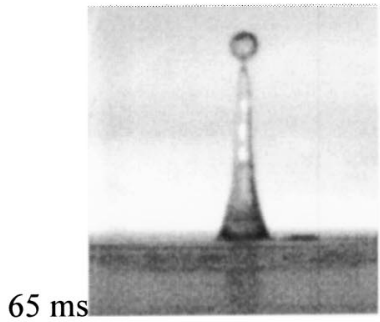

4

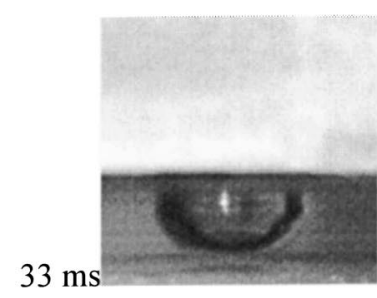

5

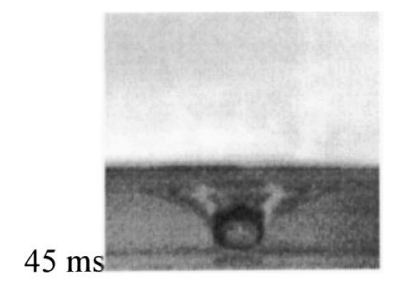

6

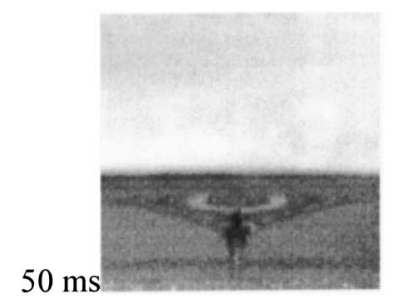

7

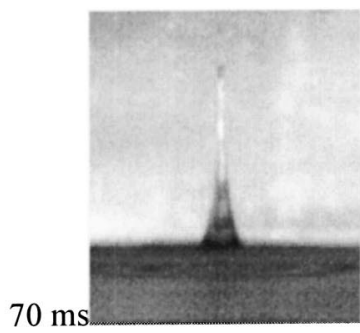

8
FIG. 3. Influence of the viscosity and depth of the target liquid on the cavity collapse and central jet parameters. For frames 1-4 (water/water) the impact conditions are $\mathrm{Fr}=158, l_{c}^{*}=1.23$, and $H^{*}=2$. For frames $5-8(\mathrm{gl} \mathrm{sol} / \mathrm{gl} \mathrm{sol})$ the impact conditions are $\mathrm{Fr}=160, l_{c}^{*}=0.91$, and $H^{*}=2$.

face of the target liquids throughout the whole lifetime.

Figure 3 offers a clearer view of how the depth and viscosity of target liquid influence the cavity collapse dynamics and the parameters of the central jet for drop impact on liquid layer. As pointed out above, the cavity collapse for drop impact on deep liquid begins with the upward motion of the cavity bottom and the cavity takes the cylindrical shape before the central jet ejection. Another situation is realized in both cases when the cavity touches (frame 1) or is about to touch (frame 5) the bottom of the liquid layers, the shape of the cavity in gl sol remains relatively smooth in contrast to the cavity shape in water (cf. frames 1 and 5 in Fig. 3), which is distorted by the capillary wave. The cavities begin to collapse from their sides, but the effect of radial focusing is weakened for water (cf. frames 2 and 6 in Fig. 3). Throughout the whole time of the collapse the cavities remain attached to the bottom of liquid layers. Thus, the closeness of the container wall prevents liquid flowing into the cavity 
from below. At the instant just before the central jets ejection, both cavities have assumed conical shapes with conical stems at their bottoms (cf. frames 3 and 7 in Fig. 3). The vertex cone angle for water is larger than that for gl sol. The cavities collapse in both cases result in the ejection of highspeed liquid jets, but the jet thickness for water is approximately double that for gl sol (cf. frames 4 and 8 in Fig. 3). In turn, the central jet speed for $\mathrm{gl}$ sol exceeds that for water. Hobbs and Osheroff ${ }^{13}$ and Macklin and Hobbs ${ }^{14}$ reported similar qualitative observations for drops impact on shallow liquid layers. Rein ${ }^{5}$ noticed similarity between thin highrising jets in the region of regular entrainment and the ones during the splash of drops on shallow liquid layers of certain depths.

From these experimental observations, a number of important conclusions follow.

In cases of high-rising jet, the cavity collapse begins from its sides and the liquid is accelerated in the radial direction toward the cavity axis of symmetry while the lowest point of the cavity remains close to its original position. Before the high-rising jet is ejected out of cavity, the cavity has assumed a conical shape. This configuration of the liquid flow resulting in a directed high-velocity mass ejection is known as Munroe effect or, in a wider sense, the cumulative effect. ${ }^{15,16}$ It should be mentioned that Rein ${ }^{5}$ also came to the similar conclusions while discussing the physical mechanisms of the thin high-speed jets ejection.

For drop impact on shallow water layers, the capillary wave propagates down the cavity wall and distorts the cavity shapes. It causes the larger cone angles at the bottom of the cavity prior to the formation of the high-speed jet. For gl sol the capillary wave on the crown wall is damped out and the formation of secondary droplets is weakened. As a result, the central jet speed for water is less than that for gl sol. In case of deep water, a cylindrical shape of the cavity is a result of a capillary wave that propagates downwards along the walls of the growing cavity. Thus, in low-viscous fluid, the interaction of capillary wave with cavity wall has strong effect on the cavity shape and, consequently, on the speed of central jets. In high viscous fluids, the influence of capillary waves is weakened. Recently, Zeff et al. ${ }^{17}$ reported similar conclusions about the role of viscosity at the collapse of a surface wave depression.

Based on the above-mentioned observations, in the next sections we will focus on the dynamic and energy formulation of drop impact phenomena concerning the cavity and central jet formation, cavity collapse, and the change of the crown angles with the liquid layer depth.

\section{DYNAMIC MODEL OF THE DROP IMPACT AND CROWN FORMATION}

As mentioned before, in cases of thin high-speed jets ejection, the cavity assumes a conical shape and the liquid accelerates inward while cavity radius decreases. Since this flow configuration is similar to that when the cumulative effect is realized we could therefore try to employ the hydrodynamic theory of cumulative jets ${ }^{15,16}$ to evaluate the central jet velocity at the cavity collapse. Following Fedorchenko

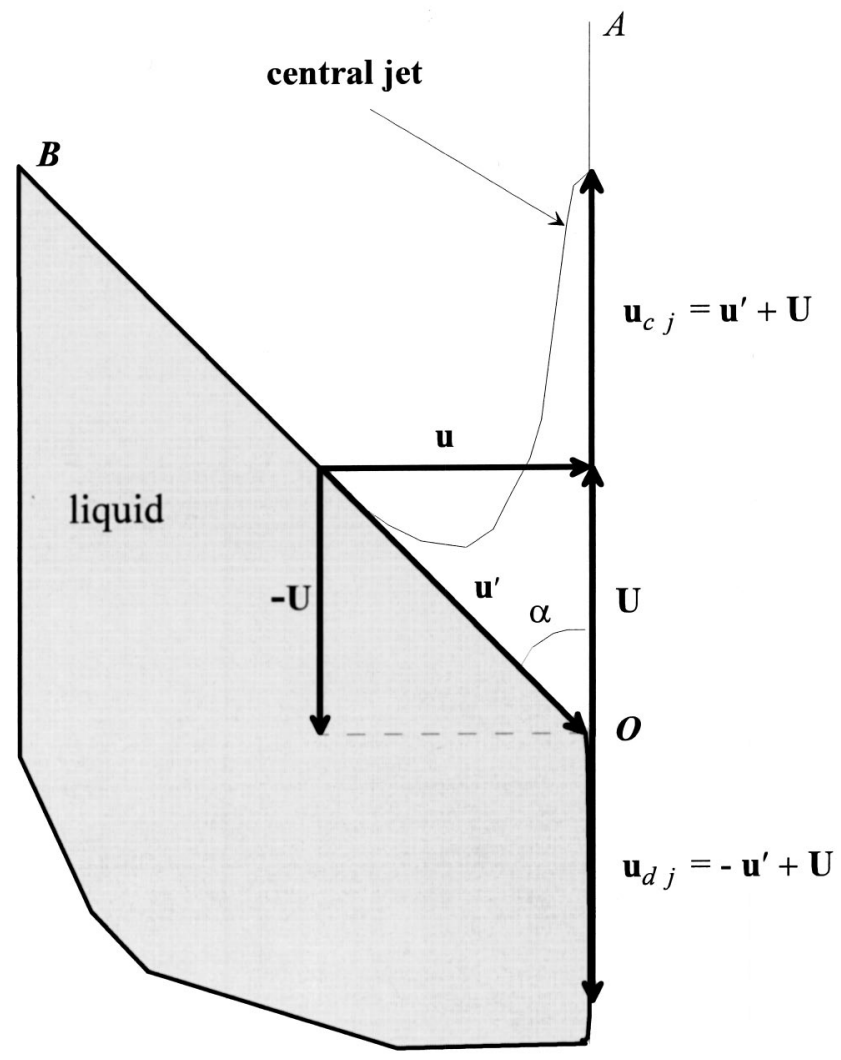

FIG. 4. Sketch of formation of upward central jet and downward one as a result of the conical cavity collapse. Line $O A$ is the axis of symmetry of the cavity; line $O B$ is the cavity wall; $2 \alpha$ is the cone vertex angle; $\mathbf{u}_{c j}$ and $\mathbf{u}_{d j}$ are the velocities of central and downward jets, respectively; $\mathbf{U}$ and $\mathbf{u}$ are the velocities of junction $O$ and cavity wall, respectively.

and Chernov, ${ }^{18}$ let us consider the collapse of the conical cavity with the vertex angle $2 \alpha$ (see Fig. 4 ). Let the cavity liquid-air boundary $O B$ have a velocity vector $\mathbf{u}$ with magnitude $u$ directed toward the axis of symmetry $O A$. In a reference frame moving with a velocity $U=u / \tan \alpha$ in which the point $O$ is immovable, the fluid velocity vector $\mathbf{u}^{\prime}$ makes an angle $\alpha$ with the axis of symmetry $O A$. In this formulation, our problem is similar to that of the oblique impact of a jet with a barrier, ${ }^{19}$ the solution of which shows that the jet at the point $O$ divides between flows upward and downward along the axis $O A$ and the flow velocities coincide with $u^{\prime}$. From the geometrical analysis, we have $u^{\prime}=u / \sin \alpha$. Passing to the stationary reference frame, it is seen that the central jet (see Fig. 4 ) has a velocity magnitude $u_{c j}$ given by

$$
u_{c j}=u^{\prime}+U=u(1+\cos \alpha) / \sin \alpha=u \cot (\alpha / 2) \text {, }
$$

while the downward jet has a velocity $u_{d j}$ given by

$$
u_{d j}=-u^{\prime}+U=-u(1-\cos \alpha) / \sin \alpha=-u \tan (\alpha / 2) .
$$

From Eqs. (3) and (4) it follows that, on the symmetry axis of the cavity, there is a fast flow of liquid moving up into the central jet and also a slow downward flow. Figure 5 shows that the $u_{c j} / u$ values increase quickly as the vertex cone angle $2 \alpha$ decreases, whereas the $u_{d j} / u$ values decreases slowly. For drop impact on shallow layer with the thickness of $H^{*}=2$, the cavity has assumed the cone shape with one- 


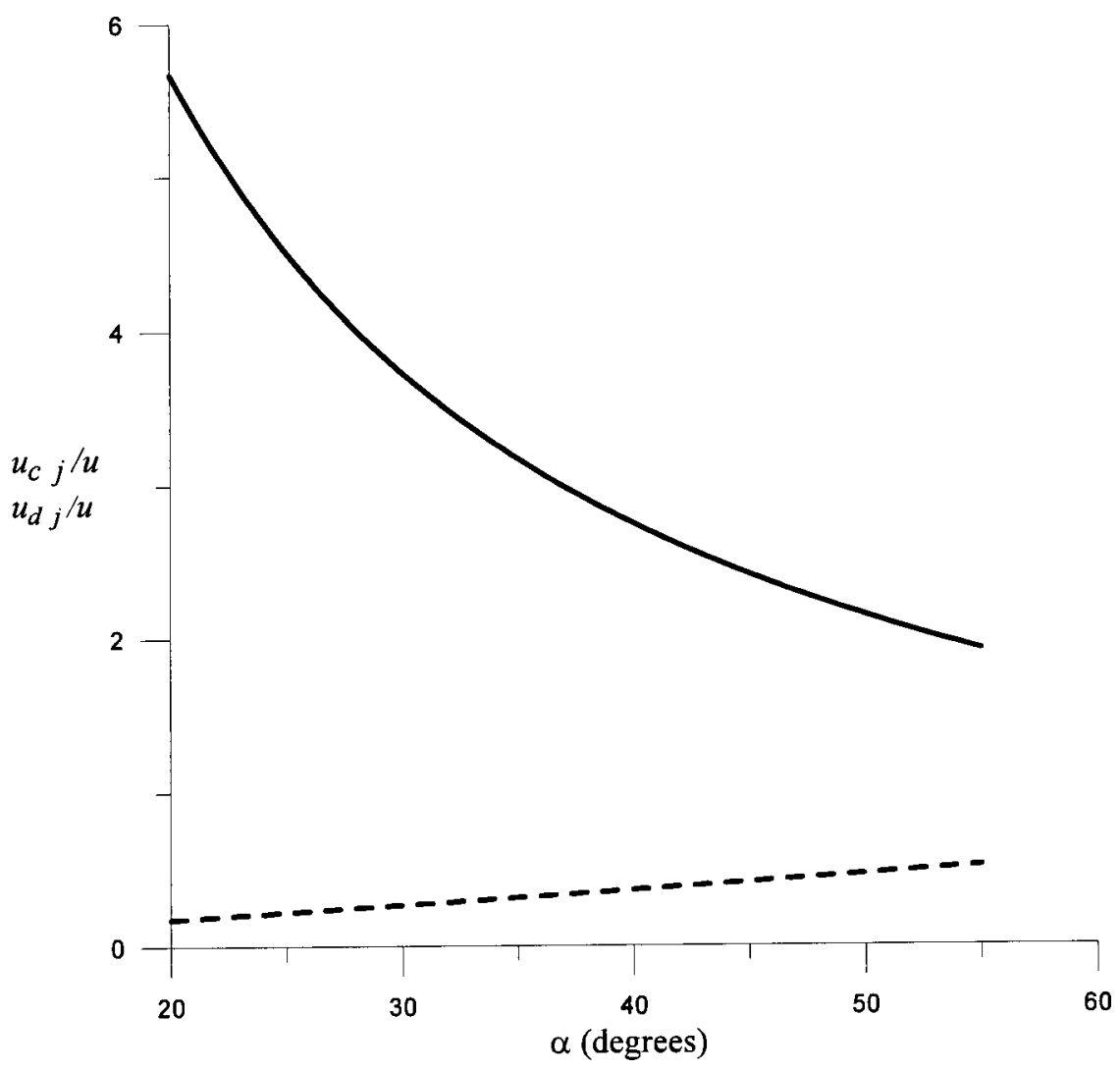

FIG. 5. Variation of $u_{c j} / u$ and $u_{d j} / u$ with the cone vertex angle $2 \alpha$.

half angle at the vertex of about $20^{\circ}$ just before jetting (see Fig. 3). From Eqs. (3) and (4), we have $u_{c j} / u=5.67$ and $u_{d j} / u=0.18$. Such a big difference between the central jet and downward flow velocities is consistent with that reported in $\operatorname{Rein}^{5}$ and our experimental observations of the impact of colored drops (not shown in this paper).

\section{A. Cavity submergence velocity}

Now we turn to the discussion of the cavity submergence velocity. Let us use a reference frame that moves towards the target liquid layer with half the initial drop velocity. In this reference frame, the target liquid layer and drop collide with each other at the same velocity of $U / 2$. Then, since the densities of the drop and the target liquid are the same, the contact point of drop/target liquid that lies on the axis of collision symmetry is stationary. Going back to the stationary reference frame, it is seen that the contact point moves at a velocity of $U / 2$. This fact allows us to find the lower value of the cavity radius as follows. In the moving reference frame, the velocity of the drop's top is $U / 2$ and the component of the drop velocity normal to the drop/target liquid interface vanishes at the interface. These boundary conditions correspond to the drop impact with the initial velocity of $U / 2$ on a solid surface. According to Fedorchenko ${ }^{20}$ and Fukai et al. ${ }^{21}$ during the initial stage of spreading, the top of the drop keeps the initial impact velocity $U / 2$. If it is assumed that the cavity reaches its maximum depth at the time $t_{m}$ when the top of drop reaches the drop/target liquid interface, then $t_{m}$ can be evaluated as $D /(U / 2)=2 D / U$. Hence the maximum cavity radius is $R_{m}=t_{m} U / 2=D$, or in the dimensionless form

$$
R_{m}^{*}=1,
$$

and the corresponding dimensionless time $T_{m}^{*}$ is

$$
T_{m}^{*}=2 \text {. }
$$

It is worthy to note that, in most experiments, we observe the drop/air interface, instead of the drop/target liquid interface. To evaluate the ratio of drop/air and drop/target liquid interfacial velocities, let us note that the cavity assumes a hemispherical shape at instant $T^{*} \approx 1$. Then, from the continuity equation in the spherical coordinates, we have

$$
R^{2} V=r^{2} \nu
$$

Here $R, V$ and $r, \nu$ are the instantaneous radius and velocity of drop/air and drop/target liquid interfaces, respectively. Assuming that the drop liquid spreads out into a thin layer over the interior of this hemisphere, we can then determine the thickness of the layer $\Delta r(\Delta r=r-R)$ from the drop's mass conservation and get

$$
\Delta r=D^{3} /\left(24 R^{2}\right) .
$$

Accounting for Eqs. (7) and (8) and the equality $r=R$ $+\Delta r$, we have

$$
V^{*} / \nu^{*}=\frac{V / U}{\nu / U}=\left(1+\frac{1}{24 R^{* 3}}\right)^{2} .
$$

Equation (9) shows that velocities $V^{*}$ and $\nu^{*}$ practically level off at $R^{*}=1$.

Since the continuity equation was applied with the liquid flowing into the crown neglected, Eq. (8) gives overestimated values of the drop liquid layer thickness and we can 


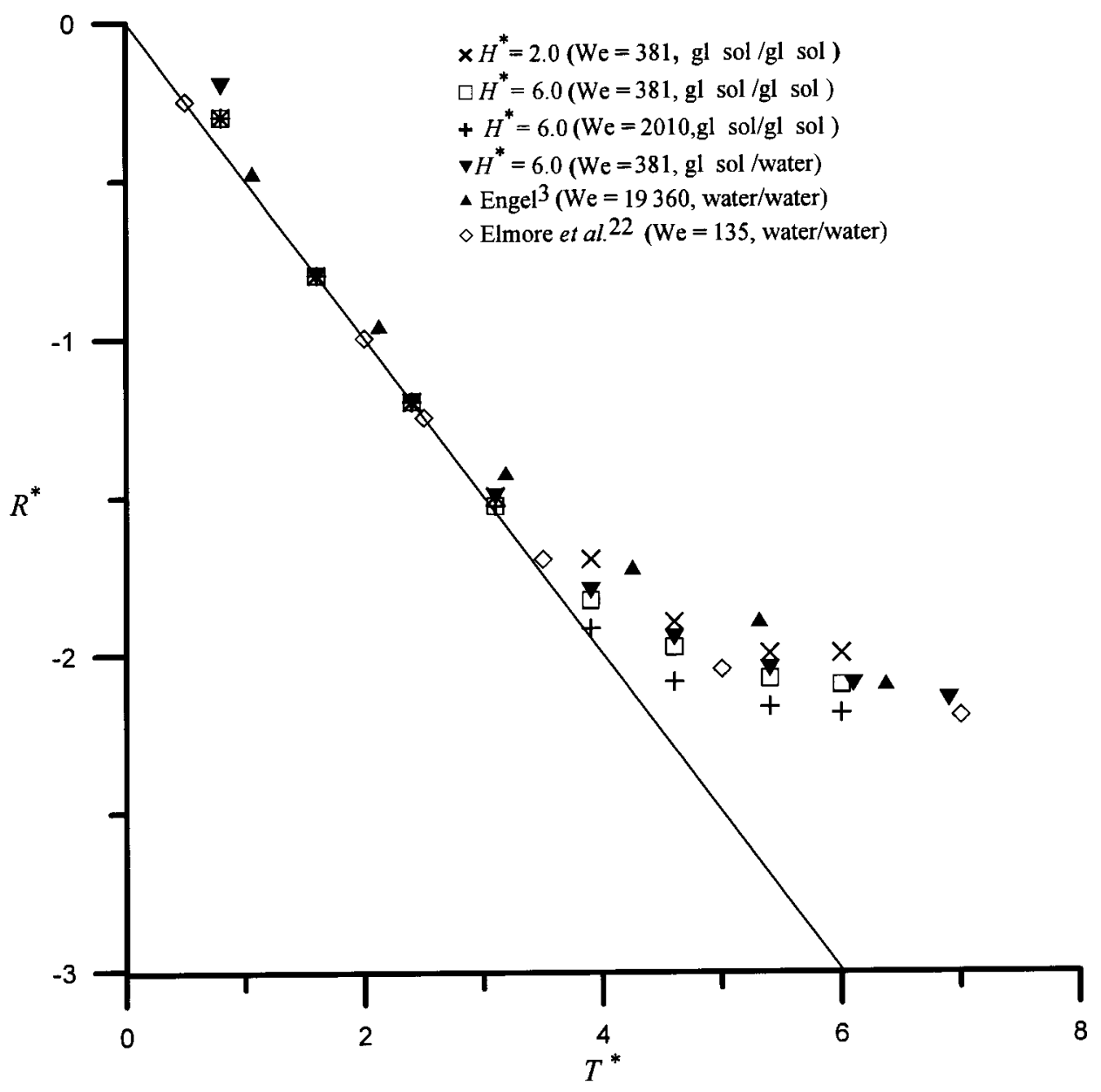

FIG. 6. Variation of the dimensionless cavity depth along the axis of symmetry with the dimensionless time for experimental data from this study and the literature. The solid line gives the theoretical prediction for the drop/ target liquid interface submergence.

be assured that the velocities level off even at the initial stage of impacts resulting in the emergence of high crowns. Figure 6 shows that, beginning from $T^{*} \approx 1$ until $T^{*} \approx 4$, the dimensionless instantaneous cavity depth $R^{*}$ is close to the linear function of the dimensionless time with a slope of $1 / 2$ for both the low viscous water and high viscous gl sol over the wide ranges of $\mathrm{We}=135-19360$ and $H^{*}=2-\infty$. Close to $T^{*}=4$, the experimental data begin to deviate from the straight line and the cavity submergence changes from the fast phase $\left(\nu^{*}=1 / 2\right)$ to the slow phase $\left(\nu^{*} \rightarrow 0\right)$ as the cavity approaches its maximum size. The experimental data of Elmore et al. ${ }^{22}$ shows the same.

\section{B. Angle of the crown}

As mentioned in Sec. III, the crown wall makes a right angle with the target liquid surface for drop impact on the target liquid layers of $H^{*}=0.5$ (see Fig. 2). The angle becomes acute for impact on thin films of $H^{*}<0.5$ (see Fig. 7).

Bearing in mind that the drop/target liquid contact point moves with a velocity $U / 2$, we can evaluate the angle between the crown wall and target liquid surface for thin layers, say $H^{*} \leqslant 0.5$. The sketch in Fig. 8 illustrates the instant when the crown emerges. Let us assume that a drop of the radius $R$ and velocity $U$ comes in touch with the target liquid surface at $t=0$. At this moment, the drop-target liquid interface begins to move downward with a velocity of $U / 2$, whereas the upper part of the drop continues moving with the initial velocity $U$. As shown in Korobkin and Pukhnachov, ${ }^{23}$ the impact of a convex body of revolution against the flat liquid surface results in a hollow circular liquid jet tangential to the body contour. Here we also assume that the crown has been thrown up tangentially to the drop contour at point $D$ (see Fig. 8) at the moment the dropliquid interface has come in touch with the solid surface at point $B$ in Fig. 8. Since angle $A O^{\prime} D$ is equal to angle $E D F$ from the geometrical relationship, the value $\beta$ of the angle $E D F$ between the tangent $C E$ to the drop contour at point $D$ and the liquid layer free surface $A F$ is given by

$$
\cos \beta=O^{\prime} A / R \text {. }
$$

At the time of $2 \mathrm{H} / \mathrm{U}$, the drop will come in touch with the solid surface at point $B$. During this period, the drop center descends a distance of $O O^{\prime}=U(2 H / U)=2 H$. Consequently, $O^{\prime} A$ is determined by the following expression:

$$
O^{\prime} A=O A-O O^{\prime}=R-2 H .
$$

Substituting Eq. (11) into Eq. (10), we have

$$
\cos \beta=1-2 H / R=1-4 H^{*} .
$$

It is important to note that, according to Eq. (12), the angle of the crown emergence depends solely on the dimensionless target liquid depth. Since the $\beta$ values vary in the range of $0 \leqslant \beta \leqslant \pi / 2$ from the physical reason, it follows that the crown wall makes a right angle to the target liquid sur- 


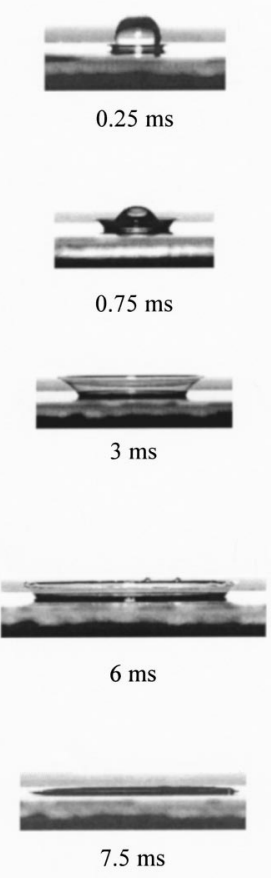

(a)
$1 \mathrm{~ms}$

$3 \mathrm{~ms}$

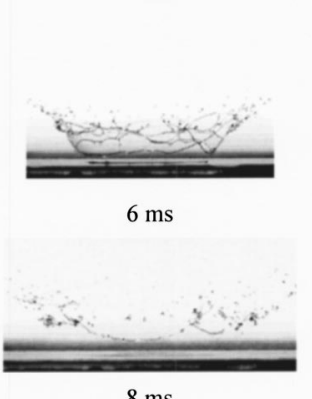

(b)
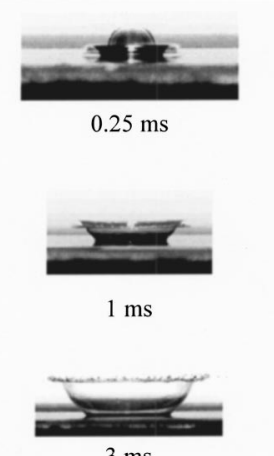

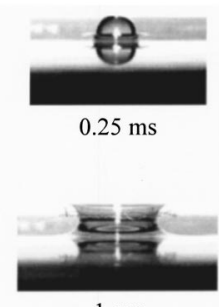

$1 \mathrm{~ms}$

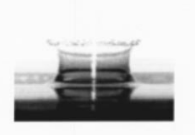

$3 \mathrm{~ms}$

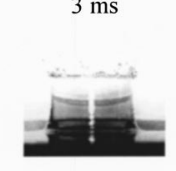

$7.5 \mathrm{~ms}$

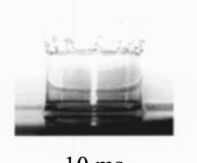

(c)
FIG. 7. Crowns emerging sequence for drop impacts ( $\mathrm{gl} \mathrm{sol} / \mathrm{gl} \mathrm{sol}$ ) on the target liquids of different thickness. (a) $H^{*}=0.05, \mathrm{We}=381, U=2.22 \mathrm{~m} / \mathrm{s}$, and $D=4.2 \mathrm{~mm}$; (b) and (c) $H^{*}=0.05$ and 0.5 , respectively, $\mathrm{We}=2010$, $U=5.1 \mathrm{~m} / \mathrm{s}$, and $D=4.2 \mathrm{~mm}$ [from Wang and Chen (Ref. 12)].

face for $H^{*} \geqslant 0.25$. Figure 7 presents the drop impacts with various impact velocities on the target liquids of different depths. The sequences in Fig. 7(c) show that drop impact at high Weber numbers results in the crown emerging at the right angle to the target liquid surface. Comparing the images in Fig. 7(c) with those in Fig. 2, it allows us to assert that the angle of the crown emergence is indeed determined only by the dimensionless target liquid depth and depends neither on drop impact velocity nor on the properties of the drop and target liquids.

Figure 9 shows that the angle $\beta$ of the crown emerging is close to $40^{\circ}$ in the cases of the drop impacts on target liquid with $H^{*}=0.05$ independently of the drop impact velocities. For $H^{*}=0.05$ and 0.5 , Eq. (12) gives $\beta$ values of $37^{\circ}$ and $90^{\circ}$, respectively. As a result, it reveals a very good consistency between the predictions and experiments.

\section{ENERGY MODEL FOR THE CAVITY AND CENTRAL JET FORMATION}

As will be shown below, Eq. (5) is the limiting solution for the maximum cavity depth and it is applicable only to the cases of the large $l_{c}^{*}$ values. To determine the cavity depth for the intermediate $l_{c}^{*}$, an energy consideration similar to that used by Engel ${ }^{2,3}$ will be applied. However, it is worth noting that we cannot use the equation derived for the maximum cavity depth by Engel ${ }^{2}$ since it is based on observations that for high-speed impacts of water drops (We $=3600-19360, \mathrm{Fr}=1255-19060)$ against water the height of the bubble approaches to the limiting value of about $2 R_{m}$. This is however not the case for our experiments (We

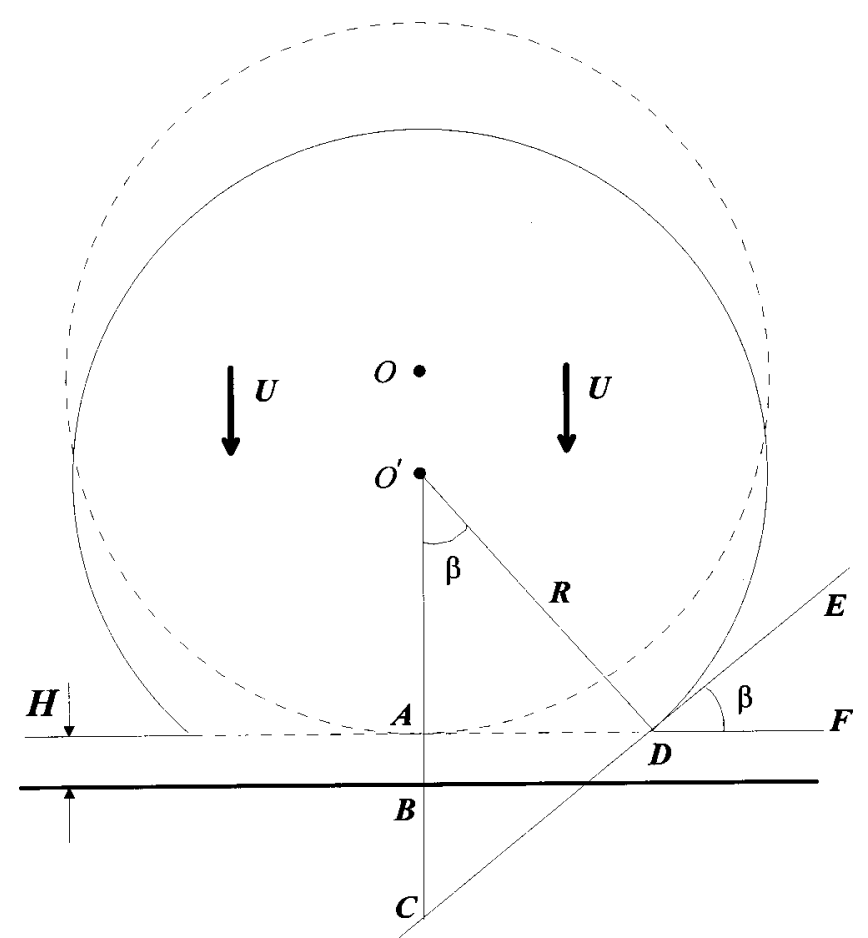

FIG. 8. Sketch of the crown emergence for drop impact on a thin liquid layer. Dashed circle is the initial position of the drop. Solid circle is the drop position at the instant when the lowest point of the drop comes in contact with the solid surface at point $B$. The straight line $C E$ is the tangent to the circle at the intersection point of the drop and the free surface of the target liquid layer. Angle $\beta$ is the angle of the crown emergence.

$=200-2500, \mathrm{Fr}=150-1050)$. Pumphrey and Elmore ${ }^{6}$ used a simplified energy consideration by assuming that the impacting drop kinetic energy is completely converted to the gravity potential energy of the cavity. But, the energy sinks, such as the potential energy of the generated surface and the kinetic energy of the secondary droplets that emanate from the upper edge of the crown [see Fig. 1(c)] have not been taken into account. As a result, the derived equation

$$
R_{m}^{*}=\left[\mathrm{Fr} /\left(3 \rho^{*}\right)\right]^{1 / 4},
$$

essentially overpredicts the experimental values of the maximum cavity depth and should also be modified for our case. Here $\rho^{*}=\rho_{t} / \rho$ and $\rho_{t}$ is the target liquid density.

As to the neglected energy sinks, the crown surface energy and the secondary drops kinetic energy, it is impossible to calculate them in terms of the drop initial parameters without invoking some empirical constants. But, since the shape of the cavity is nearly hemispherical at the time when it reaches the maximum size and the liquid around the cavity has stopped flowing, ${ }^{1,2}$ it is not difficult to calculate the cavity surface energy. By Lamb, ${ }^{24}$ the gravity potential energy of the cavity $W_{g}$ is

$$
W_{g}=\pi \rho_{t} g R_{m}^{4} / 4
$$

The surface energy of the cavity is determined by

$$
W_{s}=\sigma\left(2 \pi R_{m}^{2}-\pi R_{m}^{2}\right)=\pi \sigma R_{m}^{2} .
$$




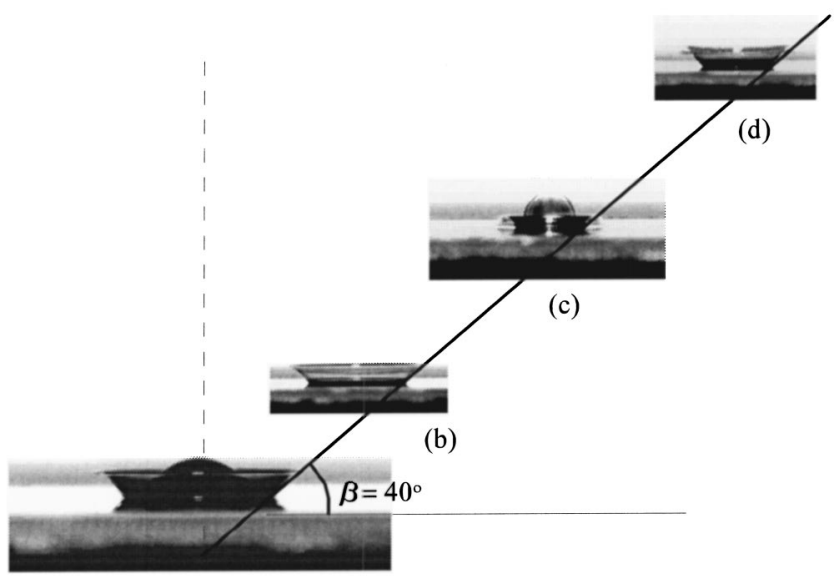

(a)

FIG. 9. The crown emerging angle $\beta$ at drop impact on the liquid film of $H^{*}=0.05$ for $\mathrm{We}=381$ and 2010 . The images correspond to the ones from Fig. 7: (a) $t=0.75 \mathrm{~ms}$ and (b) $t=3 \mathrm{~ms}$ at $\mathrm{We}=381$, (c) $t=0.25 \mathrm{~ms}$ and (d) $t=1 \mathrm{~ms}$ at $\mathrm{We}=2010$. In both cases $\beta=40^{\circ}$.

It is apparent that only if the inequality $W_{s} \ll W_{g}$ holds, the cavity surface energy can be neglected. Accounting for Eqs. (14) and (15), we can write this condition in the following form:

$$
W_{g} / W_{s}=\frac{\rho^{*}}{2}\left(\frac{R_{m}^{*}}{l_{c}^{*}}\right)^{2} \gg 1
$$

or

$$
l_{c}^{*} \ll\left(\rho^{*} / 2\right)^{1 / 2} R_{m}^{*} .
$$

It should be pointed out that the dimensionless capillary length is in fact an alternative representation of the Bond number $\mathrm{Bo}=\rho g D^{2} / \sigma=2 / l_{c}^{* 2}$. In this paper, we will use $l_{c}^{*}$ because of its direct descriptive meaning for the drop size scaling.

By examining experimental data, ${ }^{6,10}$ we come to the conclusion that Eq. (16) holds neither in Pumphrey and Elmore ${ }^{6}$ $\left(W_{g} / W_{s} \sim 1\right)$ nor in Liow ${ }^{10}\left(W_{g} / W_{s} \leqslant 1\right)$. Moreover, we noticed that, the gravity potential energy can be neglected in the cases of small droplets $\left(l_{c}^{*}>1\right)$, and only the surface energy has to be taken into consideration. Consequently, the foregoing result reveals the fact that the surface potential energy begins to prevail over the gravity potential energy during the impact process of small droplets $\left(l_{c}^{*}>1\right)$. Therefore, it would be expected that the dimensionless capillary length together with We or Fr are the main independent variables to describe completely the phenomena of drop impact on the target liquid in inviscid approximation, in particular, the cavity maximum radius. But the dimensionless capillary length has not appeared in those pertinent papers. ${ }^{6,9,10}$

\section{A. Derivation of the maximum cavity depth equation}

Let us inspect what causes this situation more deeply. In most experiments, the drop diameter, the liquid of the drop and target liquid were kept fixed, that is, $l_{c}^{*}=$ const, and only the drop impact velocity varies to cause the corresponding

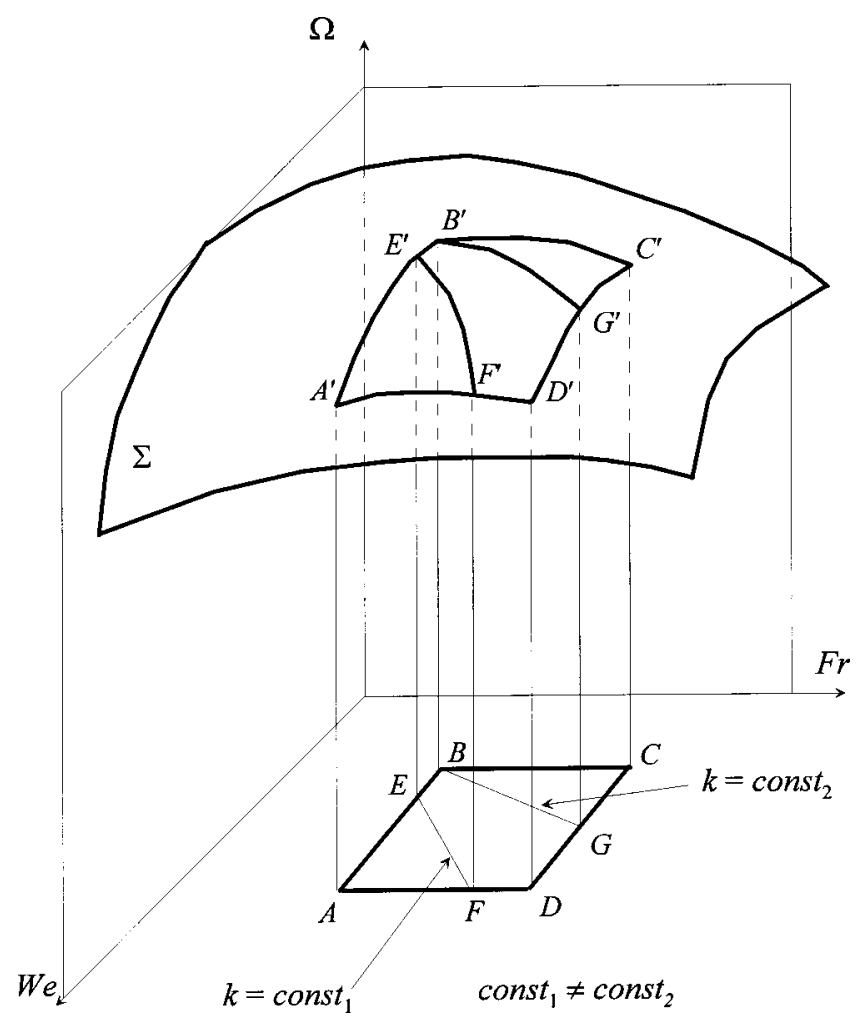

FIG. 10. Sketch of the representation of a measured quantity $\Omega$ as a function of We and Fr under the constraint $l_{c}^{*}=$ const. Each run, in the close domain $A B C D$ on the (We, Fr)-plane, is represented by a line with a constant slope of $k=2 / l_{c}^{*}$. Along lines $E F$ and $B G$, the function $\Omega\left(\mathrm{We}, \mathrm{Fr}\right.$ ) (curves $E^{\prime} F^{\prime}$ and $B^{\prime} G^{\prime}$ ) depends only on one variable (We or Fr).

change of We and Fr variables. For instance, only one experiment was carried out with the drop diameter of $5 \mathrm{~mm}$ $\left(l_{c}^{*}=0.761\right)$ from 10 reported cases in Rein. ${ }^{5}$ The drop diameters were $2.34 \mathrm{~mm} \pm 8 \%\left(l_{c}^{*}=1.63 \pm 5 \%\right)$ for the other nine cases. Applying the Buckingham Pi theorem to the problem of a drop impact on a liquid layer in the inviscid approximation, we come to the conclusion that a dimensionless measured quantity $\Omega$ (e.g., the maximum radius of the cavity) is a function of two independent variables, for instance, We and Fr. Suppose that the surface $\Sigma$ in Fig. 10 represents $\Omega$ in some region on the (We, Fr)-plane. Since there exists the relationship

$$
\mathrm{We}=\left(2 / l_{c}^{* 2}\right) \mathrm{Fr},
$$

the run under condition $l_{c}^{*}=$ const in the closed domain $A B C D$ on the (We, Fr)-plane should follow along the direct line with the slope of $k=2 / l_{c}^{* 2}=$ const. For example, if we follow along the line $E F$ in Fig. 10, we obtain the line $E^{\prime} F^{\prime}$ on the surface $\Sigma$. It is apparent that, although there seems to be two independent variables, function $\Omega(\mathrm{We}, \mathrm{Fr})$ depends only on one variable (We or Fr) along the line EF. It is not difficult to image in Fig. 10 that projections of curves $E^{\prime} F^{\prime}$ and $B^{\prime} G^{\prime}$ onto the $(\Omega, \mathrm{Fr})$-plane split up over the parameter $l_{c}^{*}$. It means that in the general case a measured quantity $\Omega$ and its partial derivative $\partial \Omega / \partial$ Fr have to be functions of both Fr and $l_{c}^{*}$. Substituting $R_{m}^{*}$ for $\Omega$, we come to the conclusion that, since in the commonly used scaling in the literature 9,10 
$R_{m}^{*}=$ const $\times \mathrm{Fr}^{n}$ the power $n$ is a constant $(n=1 / 4)$, this scaling is applicable only to the particular case of $l_{c}^{*}=0$.

Let us consider the drop impact on a deep liquid layer, in which the solid surface under the target liquid layer has no influence on the dynamics of the cavity formation and central jet eruption. We assume that the initial total energy of the drop $E_{t}$ is converted only to the gravity and surface cavity energies. It should be stressed that by doing so we are seeking the upper limit of the maximum dimensionless cavity radius. The total energy of the drop is the sum of the kinetic energy $E_{k}$ and the surface energy $E_{s}$, that is,

$$
E_{t}=E_{k}+E_{s}=\frac{\rho \pi D^{3}}{12} U^{2}+\sigma \pi D^{2} .
$$

It is convenient to rewrite Eqs. (14), (15), and (19) in the dimensionless form by using the drop surface energy as the characteristic energy scale

$$
\begin{aligned}
& E_{t}^{*}=E_{k} / E_{s}+1=\mathrm{We} / 12+1, \quad W_{g}^{*}=\frac{\rho^{*}}{2} \frac{R_{m}^{* 4}}{l_{c}^{* 2}}, \\
& W_{s}^{*}=R_{m}^{* 2} .
\end{aligned}
$$

Relating the total drop energy $E_{t}^{*}$ to the gravity energy of the cavity $W_{g}^{*}$ and the potential energy of generated surface $W_{s}^{*}$, we obtain the quartic equation in $R_{m}^{*}$,

$$
R_{m}^{* 4}+\left(2 l_{c}^{* 2} / \rho^{*}\right) R_{m}^{* 2}=\left(2 l_{c}^{* 2} / \rho^{*}\right)(\mathrm{We} / 12+1) .
$$

The solution of Eq. (21) takes the form

$$
R_{m}^{*}=\left(l_{c}^{*} / \rho^{* 1 / 2}\right)\left\{\left[1+\left(2 \rho^{*} / l_{c}^{* 2}\right)(\mathrm{We} / 12+1)\right]^{1 / 2}-1\right\}^{1 / 2} .
$$

It should be mentioned that a similar equation suggested by Prosperetti and $\mathrm{Oguz}^{9}$ was expressed through Fr and We variables. They assumed that it was possible to neglect terms $\mathrm{Fr} / \mathrm{We}$ and $(\mathrm{Fr} / \mathrm{We})^{2}$ compared to Fr and thus came to Eq. (1). However, according to Eq. (18), it is clearly that neglecting all terms of $(\mathrm{Fr} / \mathrm{We})^{m}$ is equivalent to the statement that the maximum cavity radius does not depend on $l_{c}^{*}$. This is surely not the general case in reality.

\section{B. Gravity and capillary asymptotes}

Considering Eq. (22) along the line $\mathrm{We}=$ const $\times \mathrm{Fr}$, it is easy to show that Eq. (13) is in fact the gravity asymptote of Eq. (22) and is applicable only to two particular cases: (1) $l_{c}^{*}=0$ and $\mathrm{Fr}$ is finite, and (2) $l_{c}^{*}$ is finite and $\mathrm{Fr} \rightarrow \infty$. To reveal more distinctly the behavior of $R_{m}^{*}$, let us rewrite Eq. (22) along the line $k=$ const for $\rho^{*}=1$,

$$
\begin{aligned}
& R_{m}^{*}\left(\mathrm{Fr}, l_{c}^{*}\right) \\
& \quad=\left(\frac{\mathrm{Fr}}{3}\right)^{1 / 4} \sqrt{\sqrt{1+\frac{3\left(l_{c}^{* 4}+2 l_{c}^{* 2}\right)}{\mathrm{Fr}}}-\sqrt{\frac{3}{\mathrm{Fr}} l_{c}^{* 2}} .}
\end{aligned}
$$

Figure 11(a) presents the behavior of Eq. (23) over the range of $l_{c}^{*}=0-30$ and $\mathrm{Fr}=10-10^{5}$. From this figure we notice that even at large drop $\left(l_{c}^{*}=0.8\right)$ the line $R_{m}^{*}\left(\mathrm{Fr}, l_{c}^{*}\right)$ does not have a constant slope and deviates from the gravity asymptote of Eq. (13). For $l_{c}^{*} \gg 1$, the analysis of Eq. (23) has revealed that under the condition

$$
\operatorname{Fr} /\left(6 l_{c}^{* 2}\right) \ll 1
$$

the function $R_{m}^{*}$ becomes Fr-independent and has a constant value 1 . That is, $R_{m, c}^{*}=R_{m}^{*}\left(\mathrm{Fr} \neq \infty, l_{c}^{*} \gg 1\right) \sim 1$ is the capillary asymptote of Eq. (23). The physical meaning of this fact could be explained from the following consideration. The ratio of $E_{k}$ to $E_{s}$ is equal to We/12. Accounting for Eq. (18), we obtain $E_{k} / E_{s}=\mathrm{Fr} /\left(6 l_{c}^{* 2}\right)$. This means that, while the inequality Eq. (24) holds, the kinetic drop energy $E_{k}$ remains much less than the surface one.

Figure 11(b) shows the behavior of Eq. (23) in the range of $1 \leqslant \mathrm{Fr} \leqslant 100$. Of particular interest is the functional behavior for small Froude numbers. It is important to point out that at critical point $T(\mathrm{Fr}=3)$, the function becomes $l_{c}^{*}$-independent for all drop sizes. The gravity and capillary asymptotes exchange the roles for $\mathrm{Fr}<3$. In other words, the gravity and capillary asymptotes become lower and upper boundaries, respectively. In these conditions, the process of the drop impact would be expected to proceed as the one at the state of zero gravity, ${ }^{25}$ that is, it is governed solely by capillary and inertia forces or, in other words, by the Weber number. For this case the maximum radius cavity $R_{m, \mathrm{zg}}^{*}$ is solely determined by the Weber number

$$
R_{m, \mathrm{zg}}^{*}=(1+\mathrm{We} / 12)^{1 / 2} \text {. }
$$

Here, the subscript "zg" denotes the state of zero gravity.

\section{Composed regime}

It should be mentioned here that, according to the classification of Rein, ${ }^{4}$ there exist four different characteristic regimes of the drop impact on deep liquid: floating, bouncing, coalescence and splashing which could be corresponding to different regions on (We, Fr)-variables plane. Recently, Liow $^{10}$ concluded that the regimes for floating, bouncing or coalescencing drops on the We-Fr plot are separated by lines of $\mathrm{We}=k \mathrm{Fr}$ for $0.1 \leqslant \mathrm{Fr} \leqslant 200$. But, for experiments falling in the range of $0.1 \leqslant \mathrm{Fr} \leqslant 200$, the drop impact process ceases to be a function of $\mathrm{Fr}$ and $l_{c}^{*}$ variables and begins to be a function of one variable We (or $\mathrm{Fr} / l_{c}^{* 2}$ ). In other words, all experimental events in this region have to be self-similar. As Fr increases at $\mathrm{We}=$ const the dimensionless capillary length also increases approaching to the capillary asymptote, that is, these three regimes have to coexist up to the smallest drop sizes. The upper boundary of this composed regime could be determined from the following consideration. Since this phenomenon occurs only for quite large $l_{c}^{*}$ values, on the (We, Fr)-plane this region has to lie below some line $\mathrm{We}=2 \mathrm{Fr} / l_{c}^{* 2}$. Consequently, the following inequality $\mathrm{We}<2 \mathrm{Fr} / l_{c}^{* 2}$ holds, or, $l_{c}^{* 2}<2 \mathrm{Fr} / \mathrm{We}$. The minimum value of $l_{c}^{*}$, when the capillary forces come into play, is equal to unity and we can write $\mathrm{We}<2 \mathrm{Fr}$. So, the composed region is determined by the following inequalities:

$$
l_{c}^{* 2}>1 \mathrm{We}<2 \text { Fr. }
$$



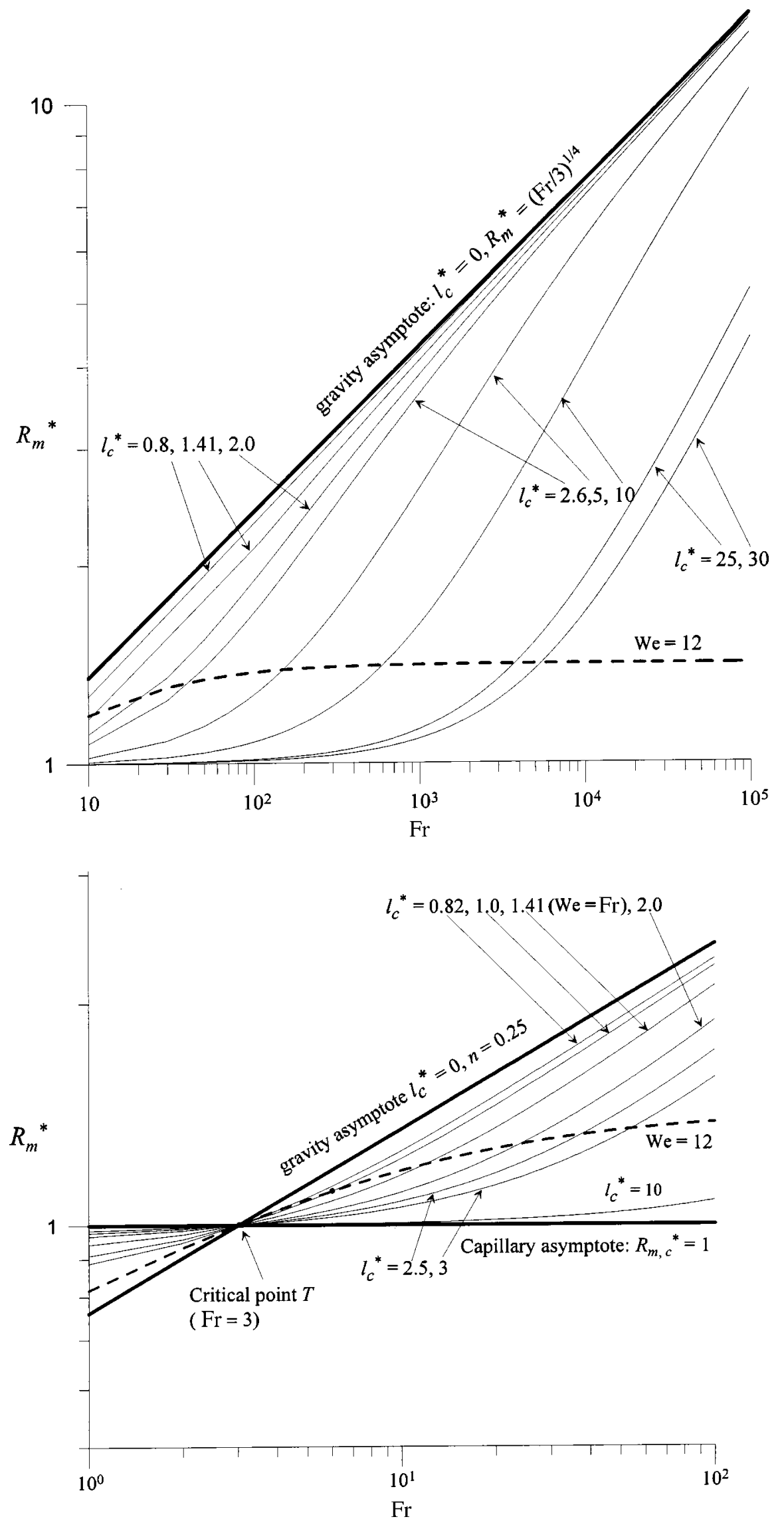

FIG. 11. The behavior of function $R_{m}^{*}\left(\mathrm{Fr}, l_{c}^{*}\right)$ along the lines $l_{c}^{*}=$ const: (a) Large Froude numbers and (b) small Froude numbers. The dashed line presents a variation of the maximum cavity depth along the curve $\mathrm{Fr}$ $=6 l_{c}^{* 2}(\mathrm{We}=12)$, that is, under the condition of $E_{k}=E_{s}$. 


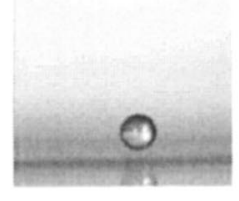

$0 \mathrm{~ms}$

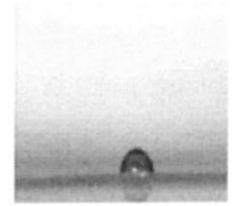

$15 \mathrm{~ms}$

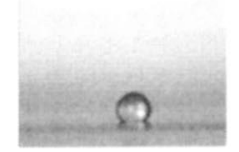

$116 \mathrm{~ms}$

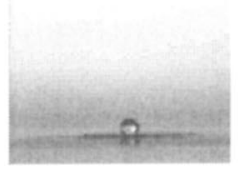

$125 \mathrm{~ms}$

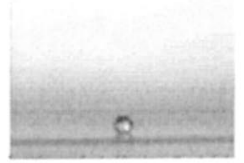

$193 \mathrm{~ms}$

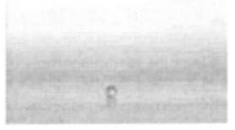

$245 \mathrm{~ms}$

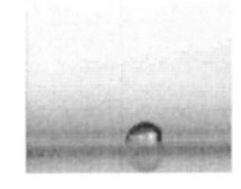

$2 \mathrm{~ms}$

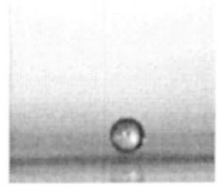

$22 \mathrm{~ms}$

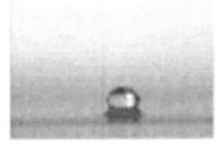

$117 \mathrm{~ms}$

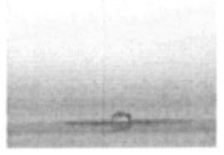

$126 \mathrm{~ms}$

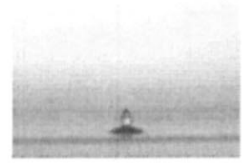

$194 \mathrm{~ms}$

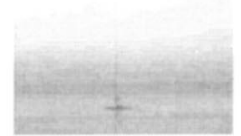

$246 \mathrm{~ms}$

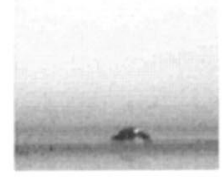

$3 \mathrm{~ms}$

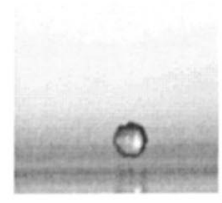

$34 \mathrm{~ms}$

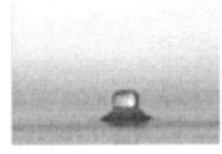

$118 \mathrm{~ms}$

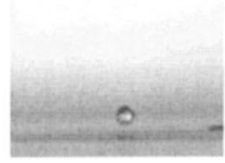

$138 \mathrm{~ms}$

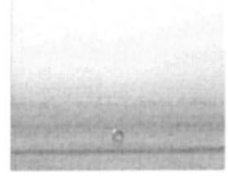

$\mathrm{D}=0.55 \mathrm{~mm}, 201 \mathrm{~ms}$

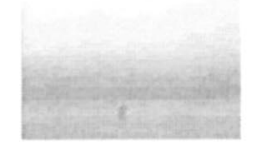

$\mathrm{D}=0.28 \mathrm{~mm}, 293 \mathrm{~ms}$

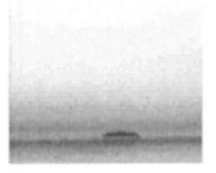

$4 \mathrm{~ms}$

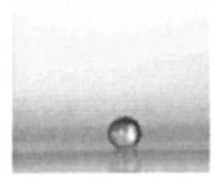

$43 \mathrm{~ms}$

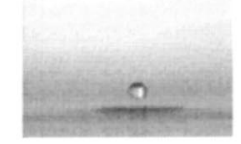

$\mathrm{D}=1.1 \mathrm{~mm}, 122 \mathrm{~ms}$

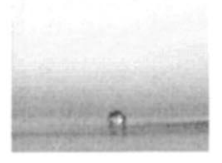

$150 \mathrm{~ms}$

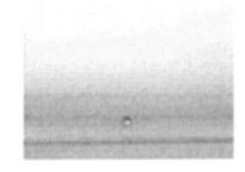

$213 \mathrm{~ms}$

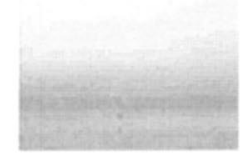

$\mathrm{D}=0.14 \mathrm{~mm}, 296 \mathrm{~ms}$
FIG. 12. The composed regime for a water/water impact. In this regime the bouncing, floating and partial coalescing coexist. The drop impact parameters at $t=0$ are $\mathrm{Fr}=14, l_{c}^{*}=1.74, D$ $=2.2 \mathrm{~mm}, U=0.55 \mathrm{~m} / \mathrm{s}$. After elapsing of 122, 201, 293, and $296 \mathrm{~ms}$ the corresponding drop diameters were found to be $1.1,0.55,0.28$, and 0.14 $\mathrm{mm}$.
In this regime the floating, bouncing and coalescing have to coexist, therefore the stated possibility ${ }^{10}$ for these processes to be separated by some lines on the We, Fr plane is open to the question. Figure 12 shows the video images of the water drop impact on water target liquid when the drop impact parameters lie in the region determined by Eqs. (26). Indeed, the floating, bouncing and partial coalescing coexist and proceed as a cascade where each step generates a smaller drop. This cascade lasted up to the smallest drop with diameter of $0.14 \mathrm{~mm}$ that was available for our optical setup. The partial drop coalescing gives rise to the daughter drop approximately one half of the parent one. The process demonstrates an intrinsic geometric and dynamic similarity and proceeds in the form of repeatable steps each consisting of the following subsequent stages: bouncing, for first step it is frames from 0 to $43 \mathrm{~ms}$; floating frames from 43 to $116 \mathrm{~ms}$; the partial coalescing with a pinching off a daughter drop frames from 117 to $122 \mathrm{~ms}$. In the experiment we observed four cascade steps which lasted for about $300 \mathrm{~ms}$. At the instant of pinch-off, the daughter drop experiences very strong shape's oscillations which gradually damp due to the effect of viscosity.

The previously reported experimental observations ${ }^{10,26}$ of the composed regimes also fall in the region determined by Eq. (26). Thoroddsen and Takehara ${ }^{26}$ observed six steps in the composed regime and suggested that it was not the limit. To evaluate the minimum drop size in the cascade (hereinafter the cutoff diameter $d_{\text {cutoff }}$ ), they conjectured that, since the characteristic velocity during the cascade process could be estimated as $d / \tau_{\sigma}$, where $d$ is the instantaneous drop diameter and $\tau_{\sigma}=\left(\rho d^{3} / \sigma\right)^{1 / 2}$ is the time scale associated with capillary oscillations of the drop, the Reynolds number $\operatorname{Re}=u d / \nu \propto d^{1 / 2}$. Therefore, for very small drops viscosity starts slowing down the partial coalescing process and destroys the similarity. It should be noted that, in the inertial oscillation region, the effect of viscous damping is deter- 


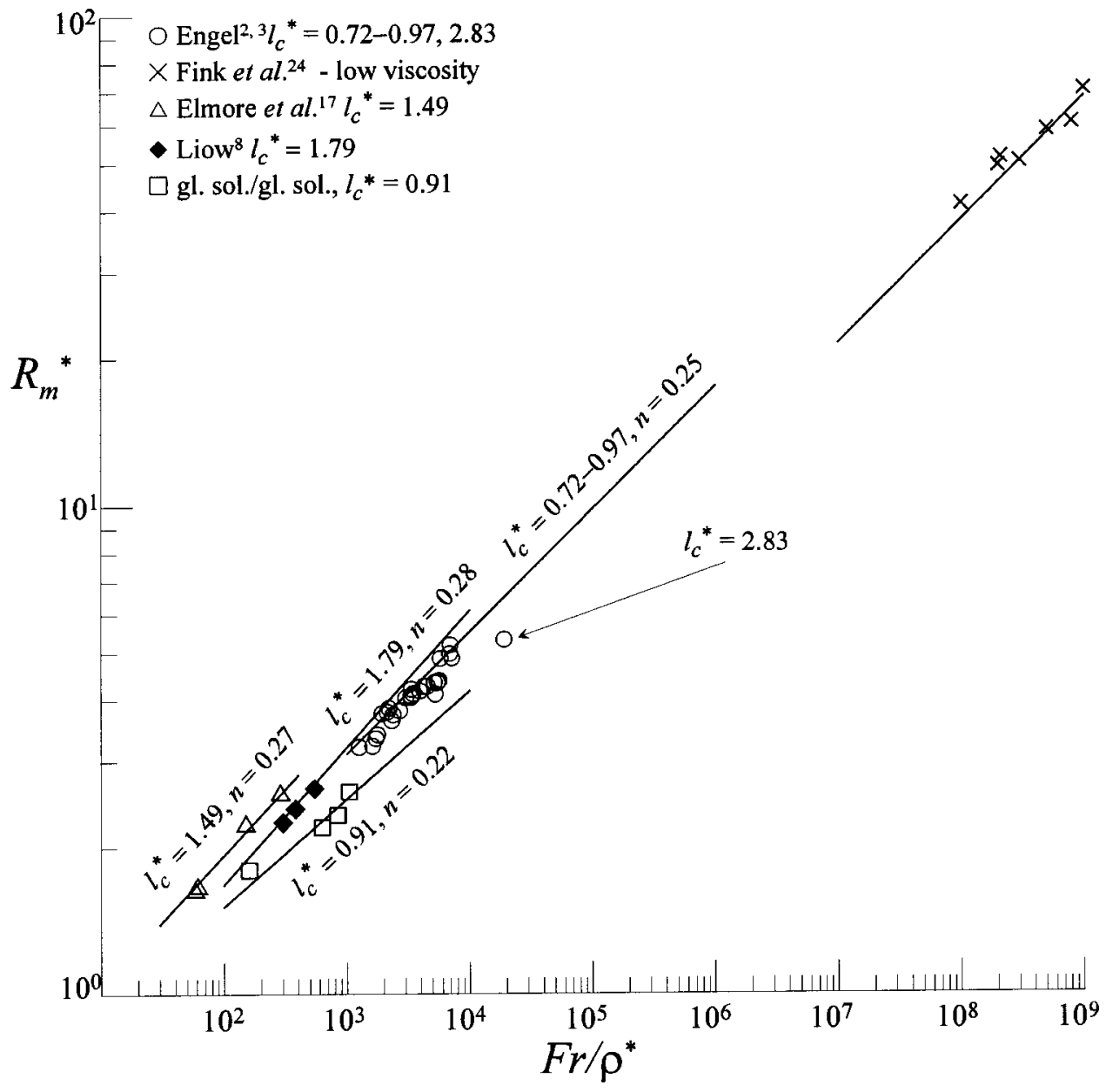

FIG. 13. Variation of the dimensionless maximum cavity depth with the Froude number along the line $l_{c}^{*}$ $=$ const. The solid lines are the theoretical predictions by means of Eq. (25). The hypervelocity data of Fink et al. (Ref. 24) for solid spheres impact into liquids is included. The dashed line is the theoretical prediction $R_{m}^{*}=0.5(\mathrm{Fr} / 3)^{1 / 4}$.

mined not by Re solely, but by the ratio of the inertial oscillation and viscous diffusion time scales, ${ }^{11,27}$ i.e., the Ohnesorge number $\mathrm{Oh}=\mu /(\rho \sigma d)^{1 / 2}$. Then the cutoff diameter $d_{\text {cutoff }}$ of the cascade process could be evaluated from the condition $\mathrm{Oh} \sim 1$. Accepting for water $\mu=0.001 \mathrm{Pas}, \rho$ $=1000 \mathrm{~kg} / \mathrm{m}^{3}, \quad \sigma=0.072 \mathrm{~N} / \mathrm{m}$ and for alcohol $\mu$ $=0.0012 \mathrm{Pas}, \quad \rho=790 \mathrm{~kg} / \mathrm{m}^{3}, \quad \sigma=0.022 \mathrm{~N} / \mathrm{m}$, we have $d_{\text {cutoff }}=1.4 \times 10^{-8} \mathrm{~m}$ for water and $d_{\text {cutoff }}=10^{-7} \mathrm{~m}$ for alcohol. It is unlikely that such small drop sizes are attainable in reality; therefore the viscous effects are hardly important in the cascade.

To estimate the cutoff diameter, we note that as discussed above at $\mathrm{Fr}=6$ and $l_{c}^{*}=1$ the kinetic and surface energies of the drop are equal to each other. Therefore, it might be expected that at $\mathrm{Fr}=6$ [see Fig. 11(b)] the change of the drop impact regimes occurs. For the smallest drop observed in our cascade We was close to 0.02 and $l_{c}^{*}$ $\approx 27.3$. At that value of the Weber number, the function $R_{m, \mathrm{zg}}^{*}$ given by Eq. (25) differs from its capillary asymptote $R_{m, c}^{*}=1$ less than $0.1 \%$, that is, $R_{m, \mathrm{zg}}^{*}$ and $R_{m, c}^{*}$ coincide practically at the smaller We. Consequently, we can assume that at some $\mathrm{We}=\mathrm{We}_{\text {cutoff }}<0.02$ the function $R_{m \text {, zg }}^{*}$ becomes We-independent. At the same time at $\mathrm{We}=\mathrm{We}_{\text {cutoff }}$ the inequality $\left(12 / \mathrm{We}_{\text {cutoff }}\right)^{1 / 2} \geqslant 27.3$ holds. From the latter we have $\mathrm{We}_{\text {cutoff }} \leqslant 0.016$. Thus, we can assume that $\mathrm{We}_{\text {cutoff }} \approx 0.015$. Now we can evaluate the dimensionless capillary length $l_{c, \text { cutoff }}^{*}$ that corresponds to the cutoff diameter as

$$
l_{c, \text { cutoff }}^{*}=\left(12 / \mathrm{We}_{\text {cutoff }}\right)^{1 / 2}=28.3 \text {. }
$$

From Eq. (27) it follows that $d_{\text {cutoff }}=0.135 \mathrm{~mm}$ for water and $d_{\text {cutoff }}=0.084 \mathrm{~mm}$ for alcohol. Since the smallest observed drop diameter in our case is $0.14 \mathrm{~mm}\left(l_{c}^{*}=27.3\right)$ and the next step of the partial coalescing should result in the drop of $0.07 \mathrm{~mm}\left(l_{c}^{*}=54.6\right)$ it is likely that $d=0.14 \mathrm{~mm}$ is the cutoff diameter. The drops of the smaller diameters completely coalesce with the receiving liquid.

\section{Maximum cavity depth}

Equation (23) allows us to break down the $\left(l_{c}^{*}, \mathrm{Fr}\right)$-variables plane into the different regions according to the gravity and capillary forces contributing to the cavity formation.

Figure 13 shows the variation of the dimensionless maximum cavity depth as a function of the Froude number for different $l_{c}^{*}$ for the data from the present study and the literature. The solid lines are the theoretical predictions from Eq. (23). Indeed, all data has splitted up over $l_{c}^{*}$ and each experimental run with $l_{c}^{*}=$ const is characterized by its own slope which depends on the value of $l_{c}^{*}$. Strictly speaking, the representation of Engel's experimental data ${ }^{2,3}$ in this plot is incorrect, since the dimensionless capillary length is not a constant but varies in the range from 0.72 to 0.97 . Therefore, although an average behavior of $R_{m}^{*}$ seems to follow the 
power law with $n=0.25$ under the gravity-dominated condition, but strong oscillations of the experimental data around the line $0.727(\mathrm{Fr} / 3)^{1 / 4}$ are evidence of the $l_{c}^{*}$ dependency. It is also apparent why the point of Engel's data ${ }^{2}$ in Fig. 13 falls away from the main group for $\mathrm{Fr}=19058$, since its corresponding $l_{c}^{*}$ value equals to 2.83 in contrast to those $l_{c}^{*}=0.72-0.97$ for the main group. Therefore, on the $\mathrm{Fr}, l_{c}^{*}$ variables plane it falls into the capillary region. Our experimental data shows that the maximum radius of the cavity is governed by Eq. (23) for all drop/target liquid combinations: water/water; water/gl sol, $\mathrm{gl} \mathrm{sol} / \mathrm{gl} \mathrm{sol}$. This reveals that the effect of the viscosity on the maximum cavity radius could be neglected.

In Fig. 13 we have included the low-viscosity results of Fink et al. ${ }^{28}$ for hypervelocity impact of solid spheres into liquid and drawn the theoretical line which fits well the experimental data. To find this line let us notice that the capillary asymptote $R_{m, c}^{*}=1$ coincides with Eq. (5) for the maximum cavity radius derived from the dynamical considerations, that is, this asymptote holds for $l_{c}^{*} \gg 1$. Keeping in mind that for high $\mathrm{Fr}$ and low $l_{c}^{*}$ the maximum cavity radius is bounded above by the gravity asymptote of Eq. (13), we come to the conclusion that the following inequality holds for any $\mathrm{Fr}$ and $l_{c}^{*}$ :

$$
1<R_{m}^{*}\left(\mathrm{Fr}, l_{c}^{*}\right)<(\mathrm{Fr} / 3)^{1 / 4} .
$$

Applying the energy model to the solid sphere impact on the target liquid, shows clearly that in this case $R_{m}^{*}$ is a function of Fr and $l_{c}^{*}$ and the maximum cavity radius obeys also Eq. (28), that is, it accepts some intermediate value between 1 and $(\mathrm{Fr} / 3)^{1 / 4}$. Then it is reasonable to write

$$
R_{m}^{*} \approx\left[(\mathrm{Fr} / 3)^{1 / 4}+1\right] / 2 \approx 0.5(\mathrm{Fr} / 3)^{1 / 4} .
$$

Figure 13 shows that Eq. (29) agrees in general with the Fink's experimental data, ${ }^{28}$ but again there are some data variations around this line, which implies that $l_{c}^{*} \neq$ const.

\section{E. Length scales of the central jet}

To find the central jet parameters, let us assume that the total energy of the impacting drop is completely converted to the central jet. The work of the jet formation includes the work $W_{g}$ against the gravitational force and the work $W_{s}$ against the surface tension force. Then, assuming the shape of the jet to be a circular cylinder having the height $h_{j}$ and the diameter $d_{j}$, we have

$$
W_{g}=\frac{\pi}{8} \rho g d_{j}^{2} h_{j}^{2}
$$

and

$$
W_{s}=\pi \sigma d_{j} h_{j} .
$$

In turn, the sum of $W_{g}$ and $W_{s}$ must be equal to the total energy of the drop from Eq. (19),

$$
E_{t}=W_{g}+W_{s} .
$$

Substituting Eqs. (19), (30), and (31) into Eq. (32), we have

$$
\frac{\pi}{8} \rho g d_{j}^{2} h_{j}^{2}+\pi \sigma d_{j} h_{j}=\frac{\rho \pi D^{3}}{12} U^{2}+\sigma \pi D^{2} .
$$

Introducing a new variable $Z=d_{j}^{*} h_{j}^{*}$, Eq. (33) becomes

$$
Z^{2}+4 l_{c}^{* 2} Z-4 l_{c}^{* 2}(\mathrm{We} / 12+1)=0
$$

Choosing the positive sign of the root, we have

$$
Z=d_{j}^{*} h_{j}^{*}=2 l_{c}^{* 2}\left\{\left[1+(\mathrm{We} / 12+1) / l_{c}^{* 2}\right]^{1 / 2}-1\right\} .
$$

Physically, the parameter $Z$ determines a dimensionless area of the central jet surface. From Eq. (35) it follows that by using only the energy considerations, it is impossible to find both the jet diameter and its height. However, once we know the diameter of the central jet, for example, from an experiment, then Eq. (35) determines uniquely the maximum height of the central jet.

Rewriting Eq. (35) along the line $l_{c}^{*}=$ const, we obtain

$$
Z=2\left[\sqrt{\left(l_{c}^{* 2}+\frac{1}{2}\right)^{2}+\frac{2 \mathrm{Fr}-3}{12}}-l_{c}^{* 2}\right] .
$$

From Eq. (36) the gravity $Z_{g}\left(l_{c}^{*}=0\right)$ and capillary $Z_{c}\left(l_{c}^{*}\right.$ $\rightarrow \infty$ ) asymptotes can be derived as

$$
Z_{g}=(2 \mathrm{Fr} / 3)^{1 / 2} \text { and } Z_{c}=1 \text {. }
$$

Figure 14 illustrates the change of $Z$ with Fr along the line $l_{c}^{*}=$ const. A comparison between this figure and Fig. 11 shows the similarity in the behavior of $Z$ and $R_{m}^{*}$ on $\left(\mathrm{Fr}, l_{c}^{*}\right)$-coordinates plane.

For two cases depicted in Fig. 3 now we can show that indeed the energy density of central jet for $\mathrm{gl} \mathrm{sol} / \mathrm{gl} \mathrm{sol} \mathrm{im-}$ pact exceeds that for water/water impact. To this end, let us define the energy density $e_{j}$ of the central jet

$$
e_{j}=E_{t} / \mathrm{Vol}_{j}=4 E_{t} /\left(\pi d_{j}^{2} h_{j}\right),
$$

or, in the dimensionless form

$$
e_{j}^{*}=e_{j} \operatorname{Vol}_{d} / E_{t}=1 /\left(3 d_{j}^{* 2} h_{j}^{*}\right),
$$

where $\operatorname{Vol}_{j}=\pi d_{j}^{2} h_{j} / 4$ is the volume of central jet, $\operatorname{Vol}_{d}$ $=\pi D^{3} / 6$ is the volume of drop. For water/water and $\mathrm{gl} \mathrm{sol} /$ gl sol impacts we have from Eq. (35) $Z_{1}=d_{j 1}^{*} h_{j 1}^{*}=8$ and $Z_{2}=d_{j 2}^{*} h_{j 2}^{*}=9$, respectively. Accounting for Eq. (39), the ratio $e_{j 1}^{*} / e_{j 2}^{*}$ takes the form

$$
e_{j 1}^{*} / e_{j 2}^{*}=9 d_{j 2}^{*} /\left(8 d_{j 1}^{*}\right) .
$$

Figure 3 shows that the diameter of the central jet for water/ water impact is about twice as big as that for $\mathrm{gl} \mathrm{sol} / \mathrm{gl} \mathrm{sol}$ impact, that is, we can assume $d_{j 1}=2 d_{j 2}$, or, $d_{j 1}^{*}$ $=2 d_{j 2} / D_{1}$ and $d_{j 2}^{*}=d_{j 2} / D_{2}$. Here $D_{1}=3.12 \mathrm{~mm}$ and $D_{2}$ $=3.64 \mathrm{~mm}$ are the drops diameters in the first and second cases, respectively. Substituting $d_{j 1}^{*}=2 d_{j 2} / 3.12$ and $d_{j 2}^{*}$ $=d_{j 2} / 3.64$ in Eq. (40) we obtain $e_{j 1}^{*} / e_{j 2}^{*}=0.43$. Thus, the energy density of the central jet for $\mathrm{gl} \mathrm{sol} / \mathrm{gl} \mathrm{sol} \mathrm{impact} \mathrm{is}$ more than two times higher than that for water/water impact.

In case of the gl sol large drops impact on gl sol deep liquid layers $\left(H^{*}=6\right)$ the central jets reach their greatest heights without pinching off the secondary droplets and assume a shape close to cylindrical at this instant. Therefore, we could try to apply our theory to this case. As noted above, 


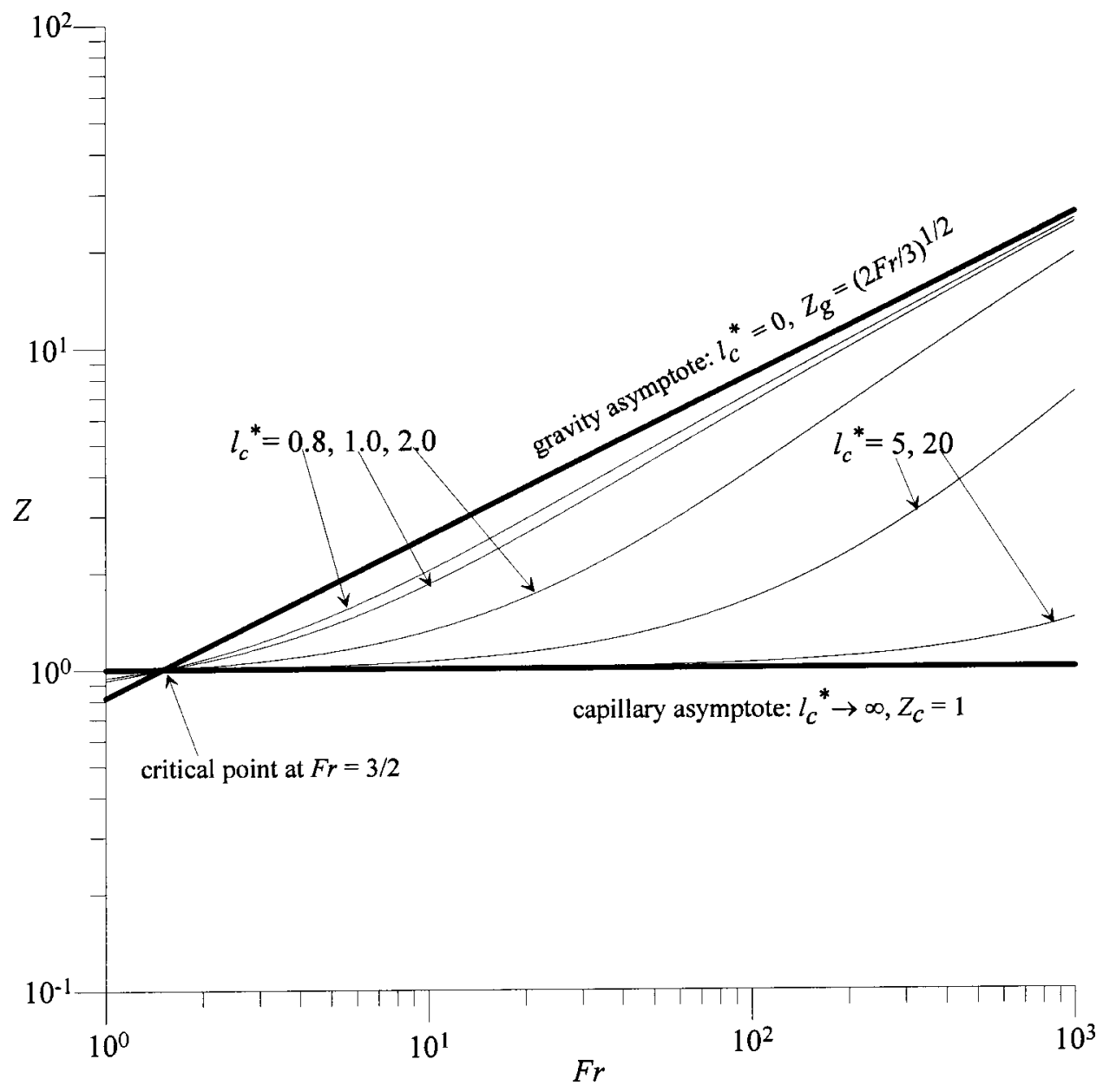

FIG. 14. Variation of the dimensionless surface area of the central jet $Z$ with the Froude number along the line $l_{c}^{*}=$ const. The gravity asymptote $\left(l_{c}^{*}\right.$ $=0)$ is $Z_{g}=(2 \mathrm{Fr} / 3)^{1 / 2}$, the capillary asymptote $\left(l_{c}^{*}=\infty\right)$ is $Z_{c}=1$, the critical point is $\mathrm{Fr}=3 / 2$.

from the theory we can find only parameter $Z=d_{j}^{*} h_{j}^{*}$. To determine both $d_{j}^{*}$ and $h_{j}^{*}$ we have to find an additional equation in which $d_{j}^{*}$ and $h_{j}^{*}$ are related to each other. For the unsteady motion of a gas cavity in an ideal fluid, the induced mass is half the mass of the fluid displaced by the cavity. ${ }^{11}$ As a first approximation, we can consider the cavity collapse as an unsteady motion of a sphere of radius $R_{m}^{*}$ in an ideal fluid. In this case the induced mass $M_{i}$ is

$$
M_{i}=2 \pi \rho R_{m}^{* 3} / 3 \text {. }
$$

If we assume that this mass forms the central jet, we obtain

$$
2 R_{m}^{* 3} / 3=d_{j}^{* 2} h_{j}^{*} / 4 .
$$

As we consider impact of large drops with $l_{c}^{*}<1$, we can substitute the gravity asymptotes Eqs. (1) and (37) for $R_{m}^{*}$ and $d_{j}^{*} h_{j}^{*}$. As a result, we find

$$
\begin{aligned}
& h_{j}^{*}=1.43 \mathrm{Fr}^{1 / 4}, \\
& d_{j}^{*}=0.57 \mathrm{Fr}^{1 / 4} .
\end{aligned}
$$

Figure 15 does show that height of the central jet agrees well with the theoretical prediction given by Eq. (43).

From Eqs. (1), (43), and (44), it follows that the characteristic linear scale $L^{*}$ of a fluid flow and the Froude number are related by the power law $L^{*} \sim \mathrm{Fr}^{1 / 4}$ if the fluid flow is gravity driven.

\section{CAPILLARY-GRAVITY DRIVEN CAVITY COLLAPSE}

To evaluate the temporal scales of the cavity collapse, let us consider the capillary-gravity driven collapse of the hemisphere cavity with the initial radius $R_{m}$. We assume that the target liquid of the density $\rho_{t}$ and an excessively thin layer of the original drop liquid over the surface of this hemisphere are immovable at $t=0$. Following the standard technique ${ }^{11}$ to solve such kind of problems, in the approximation of the inviscid spherically symmetric collapse we find the dimensionless cavity collapse time $T_{c}^{*}$ as

$$
\begin{aligned}
T_{c}^{*}= & \sqrt{\frac{\rho^{*} \mathrm{We}}{2}} R_{m}^{* 3 / 2} \\
& \times \int_{0}^{1}\left[\frac{1}{z^{3}}-\frac{1}{z}+\frac{\rho^{*}}{2 l_{c}^{* 2}} R_{m}^{* 2}\left(\frac{1}{z^{3}}-z\right)\right]^{-1 / 2} d z,
\end{aligned}
$$

where $z=R / R_{m}, R$ is the instantaneous radius of the cavity.

As in the case of the maximum cavity radius, $T_{c}^{*}$ is also a function of We or Fr along the line $l_{c}^{*}=$ const. From Eqs. (13), (18), and (45), the gravity $T_{c g}^{*}\left(l_{c}^{*} \rightarrow 0\right)$ and capillary $T_{c c}^{*}$ asymptotes $\left(l_{c}^{*} \rightarrow \infty\right)$ become

$$
\begin{aligned}
& T_{c g}^{*}=0.832 \mathrm{Fr}^{5 / 8}, \\
& T_{c c}^{*}=0.618 \mathrm{We}^{1 / 2} .
\end{aligned}
$$




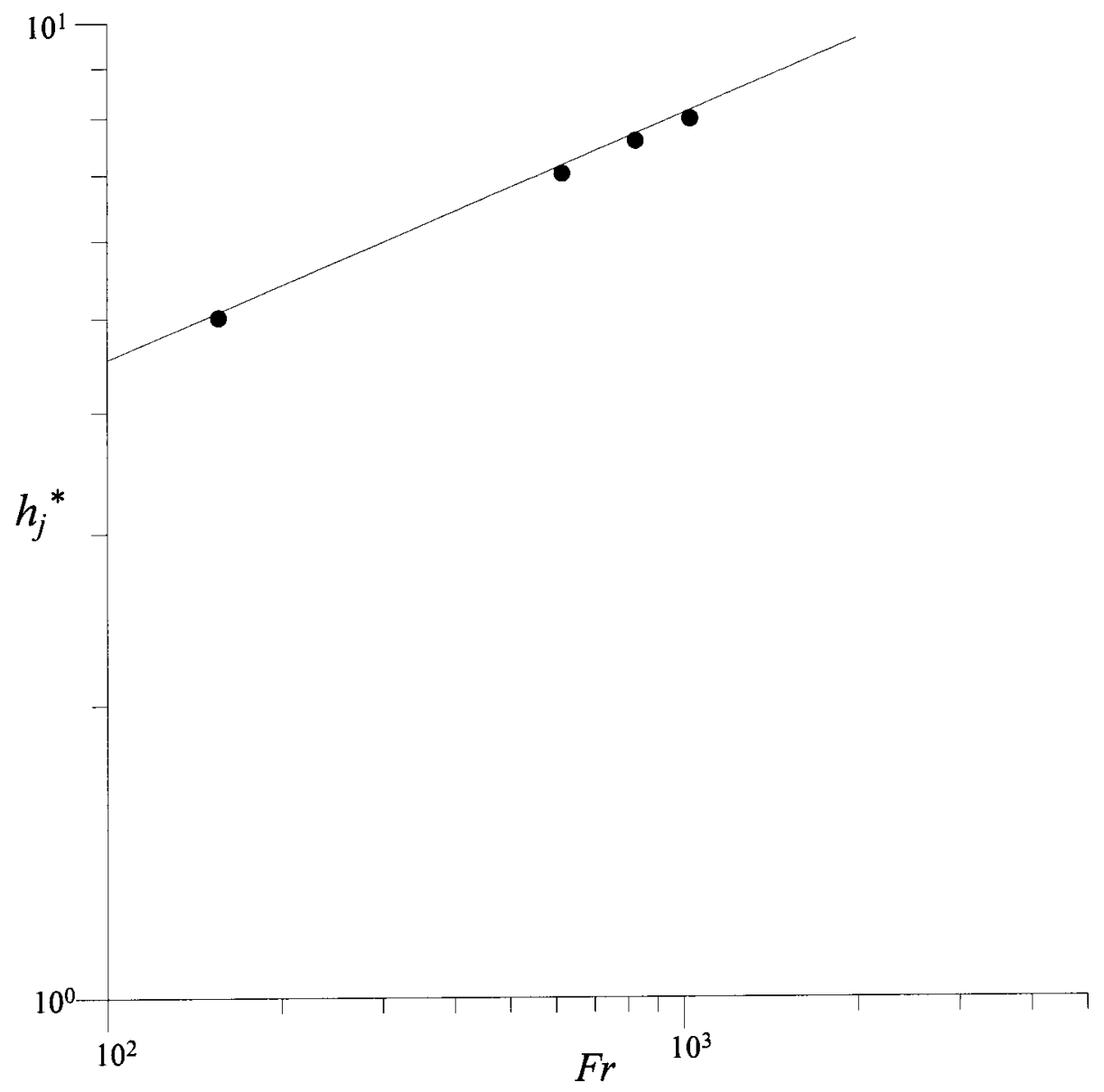

FIG. 15. Variation of the maximum central jet height with the Froude number. The solid line is the theoretical prediction $h_{j}^{*}=1.43 \mathrm{Fr}^{1 / 4}$. The circles are experimental data from our study (gl sol/gl sol impact, $H^{*}=6$ ).
The characteristic velocity scale $V_{c}^{*}$ for the cavity collapse can be evaluated as $V_{c}^{*}=R_{m}^{*} / T_{c}^{*}$. In the gravity- and capillary-dominated cases, we have

$$
\begin{aligned}
& V_{c g}^{*}=0.913 \mathrm{Fr}^{-3 / 8}, \\
& V_{c c}^{*}=1.618 \mathrm{We}^{-1 / 2},
\end{aligned}
$$

respectively.

Substituting Eq. (23) into Eq. (45), we can evaluate the cavity collapse time for any particular drop impact regime. But of prime importance is the cavity collapse time along the line $l_{c}^{*}=$ const. Figure 16 presents two such cases for data from our study and one case for data from Liow. ${ }^{10}$ It is important to note that both the data for high-viscous gl sol and low-viscous water are predicted well by Eq. (45). As $l_{c}^{*}$ decreases, the curves $T_{c}^{*}\left(\mathrm{Fr}, l_{c}^{*}=\right.$ const $)$ approach the gravity asymptote of Eq. (46).

To explain the experimental observations why the drop impacts result in a thick slow central jet for relatively high Froude numbers in the gravity dominated cases, let us write the expression for the dimensionless central jet velocity by combining Eqs. (3) and (48):

$$
u_{c j}^{*}=0.913 \mathrm{Fr}^{-3 / 8} \cot (\alpha / 2) \text {. }
$$

Figure 17 shows the dimensionless central jet velocity as a function of the Froude number for different values of the angle $\alpha$. As is seen from Fig. 17, for all $\alpha$ values the central jet velocity remains smaller than that of the drop impact and decreases as the Froude number increases. The central jet velocity changes weakly over a wide range of Froude numbers for larger $\alpha$-angles. This trend is consistent with our experimental observations for large drops impacting on deep liquid layers.

\section{CONCLUSIONS}

In the present work new experimental observations are provided for drops impact on liquid layers in the region of fully developed splash in wide range of drop impact parameters. Based on experimental observations a dynamical model describing the cavity submergence, the central jet and crown ejection has been suggested. The model predicts a constant speed of the drop/target liquid interface submergence independently of viscosities of drop and target liquid, which is one-half of the initial drop impact velocity. For the conical cavity collapse it has been shown that a slow downward jet always accompanies the high-speed central jet. The central jet velocity increases as the vertex angle of the conical cavity decreases, whereas the downward jet velocity decreases. The model allows us to determine the angle between the crown wall and the target liquid surface at drops impact on shallow liquid layers. As has been shown, the angle of the crown emergence depends solely on the dimensionless target liquid depth $H^{*}$ : at $H^{*} \geqslant 0.25$ the angle is constant and equals to $90^{\circ}$, at $H^{*}<0.25$ the angle is acute and monotoni- 

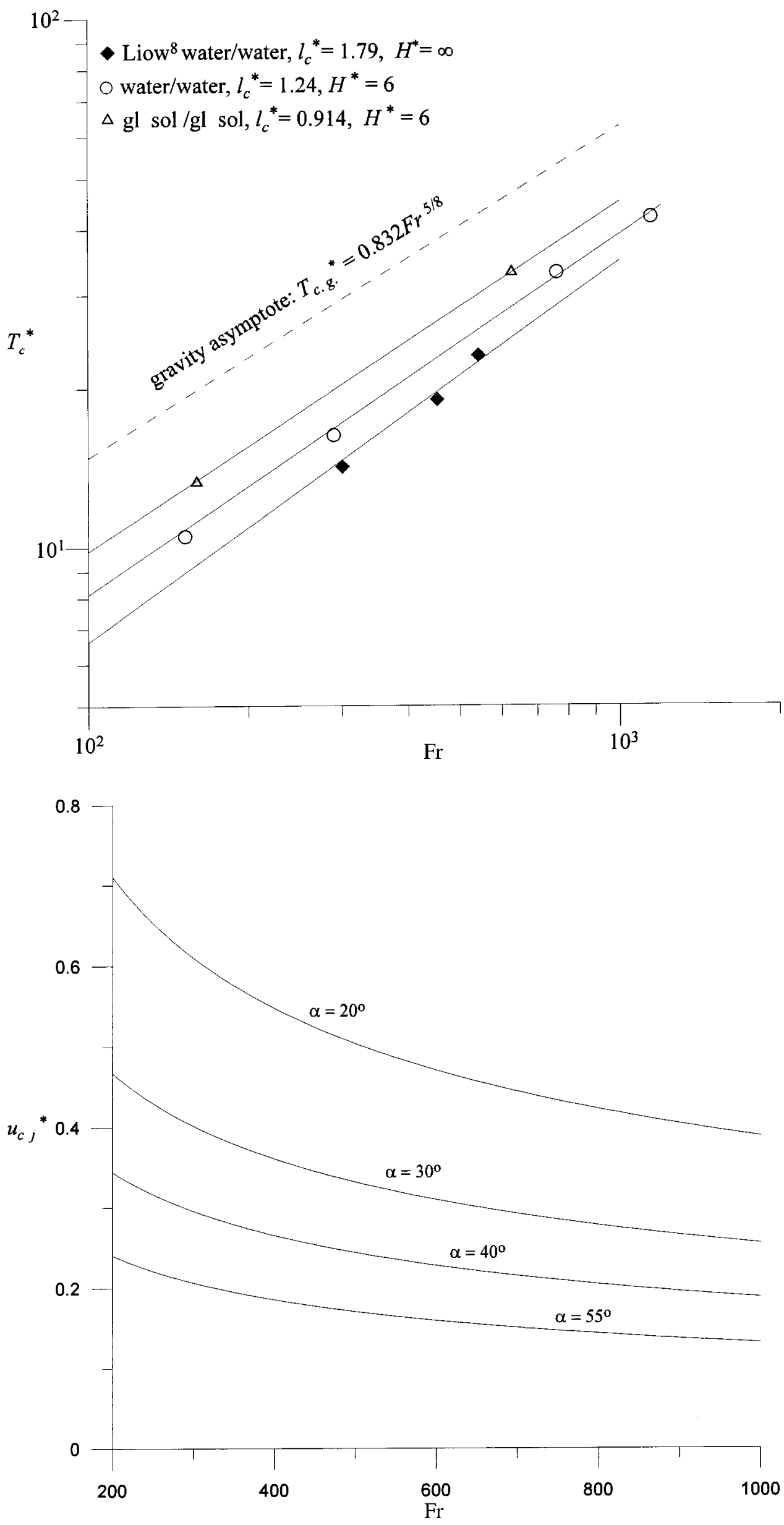

FIG. 16. Variation of the cavity collapse time with the Froude number at $l_{c}^{*}=$ const for experimental data from this study and the literature. The dashed line is the gravity asymptote $T_{c g}^{*}=0.832 \mathrm{Fr}^{5 / 8}$, and solid lines are theoretical predictions by means of Eqs. (47).

FIG. 17. The dimensionless central jet velocity in the gravity-dominated case as a function of the Froude number for different cone angles $2 \alpha$. 
cally decreases with $H^{*}$. All theoretical predictions agree well with data from this study and the literature.

Based on an energy consideration theoretical models for the maximum cavity depth and central jet formation have been suggested. These models show that the maximum depth of the cavity and parameters of the central jets depend on both the Froude number and dimensionless capillary length. As it follows from the models, the scaling $\mathrm{Fr}^{1 / 4}$ is the gravity asymptote and it is applicable only to two particular cases: (1) $l_{c}^{*}=0$ and $\mathrm{Fr}$ is finite, (2) $l_{c}^{*}$ is finite and $\mathrm{Fr}=\infty$. In the general case, the scaling $\mathrm{Fr}^{n}$ is applicable only in the narrow range of the Froude number and the power $n$ depends on $l_{c}^{*}$. The theoretical analysis and experimental evidence reveal that in the region $l_{c}^{* 2}>1$ or $\mathrm{We}<2 \mathrm{Fr}$ and $0.1<\mathrm{Fr}<200$ there exists the composed regime. In this regime the floating, bouncing, and coalescing coexist and proceed as a cascade where each step generates a smaller drop. The critical value of $l_{c}^{*}$ has been found to be 28.3, which corresponds to 0.135 $\mathrm{mm}$ and $0.084 \mathrm{~mm}$ for water and alcohol drop diameters, respectively. After drop has reached this critical size the cascade ceases as the drop coalesces with the receiving liquid.

The analytical solution for the capillary-gravity driven semispherical cavity collapse has been found. Theoretical predictions of the cavity collapse time agree well with experimental data from this study and the literature. From the solution it follows that the gravity $\left(l_{c}^{*} \rightarrow 0\right)$ and capillary asymptotes $\left(l_{c}^{*} \rightarrow \infty\right)$ are described by the power functions $\mathrm{Fr}^{5 / 8}$ and $\mathrm{We}^{1 / 2}$, respectively. At the conical cavity collapse the central jet velocity depends on the vertex angle of the cone $\alpha$. In the gravity-dominated case the central jet velocity depends on $\mathrm{Fr}$ and $\alpha$ as $\mathrm{Fr}^{-3 / 8} \cot (\alpha / 2)$.

Our experiments with drop impact on shallow liquid layers have shown that the highest jets occur for depth $H^{*}=2$ for both water/water and $\mathrm{gl} \mathrm{sol} / \mathrm{gl}$ sol impacts. At this depth the cavity at its maximum extension touches or is about to touch the bottom of the liquid layer. The cavity assumes the conical shape shortly before the central jet ejection. The speed of central jet for $\mathrm{gl} \mathrm{sol} / \mathrm{gl} \mathrm{sol}$ impact is larger than that for water/water impact. The reason is that for water/water impact the capillary wave disturbs the cavity shape causing the angle $\alpha$ to increase. For $\mathrm{gl} \mathrm{sol} / \mathrm{gl}$ sol impact the capillary wave is damped due to high viscosity of the liquid.

\section{ACKNOWLEDGMENTS}

The authors thank Professor K.-C. Wu for valuable comments on this paper. Partial experimental support from C.-H. Hsueh, M.-H. Wu, L.-W. Chien, and S.-S. Chen is gratefully acknowledged. This work was supported by the National Science Council, Taiwan, Republic of China under Grant No. NSC 89-2811-E-002-0074.

${ }^{1}$ A. M. Worthington, A Study of Splashes (Longmans, Green and Co., London, 1908).
${ }^{2}$ O. G. Engel, "Crater depth in fluid impacts," J. Appl. Phys. 37, 1798 (1966).

${ }^{3}$ O. G. Engel, "Initial pressure, initial flow velocity, and the time dependence of crater depth in fluid impacts," J. Appl. Phys. 38, 3935 (1967).

${ }^{4}$ M. Rein, "Phenomena of liquid drop impact on solid and liquid surfaces," Fluid Dyn. Res. 12, 61 (1993).

${ }^{5} \mathrm{M}$. Rein, "The transition regime between coalescing and splashing drop," J. Fluid Mech. 306, 145 (1996).

${ }^{6}$ H. C. Pumphrey and P. A. Elmore, "The entrainment by drops impact," J. Fluid Mech. 220, 539 (1990).

${ }^{7}$ D. Morton, M. Rudman, and J. L. Liow, "An investigation of the flow regimes resulting from splashing drops," Phys. Fluids 12, 747 (2000).

${ }^{8}$ J. Shin and T. A. McMahon, "The tuning of a splash," Phys. Fluids A 2, $1312(1990)$.

${ }^{9}$ A. Prosperetti and H. N. Oguz, "The impact of drops on liquid surfaces and the underwater noise of rain," Annu. Rev. Fluid Mech. 25, 577 (1993).

${ }^{10}$ J. L. Liow, "Splash formation by spherical drops," J. Fluid Mech. 427, 75 (2001).

${ }^{11}$ L. D. Landau and E. M. Lifshitz, Fluid Mechanics (Pergamon, Oxford, England, 1987).

${ }^{12}$ A.-B. Wang and C.-C. Chen, "Splashing impact of a single drop onto very thin liquid films," Phys. Fluids 12, 2155 (2000).

${ }^{13}$ P. V. Hobbs and T. Osheroff, "Splashing drops on shallow liquids," Science 158, 1184 (1967).

${ }^{14}$ W. C. Macklin and P. V. Hobbs, "Subsurface phenomena and splashing of drops on shallow liquids," Science 166, 107 (1969).

${ }^{15}$ G. Birkhoff, D. P. MacDougall, E. M. Pugh, and G. Taylor, "Explosive with lined cavities," J. Appl. Phys. 19, 563 (1948).

${ }^{16}$ M. A. Lavrentyev and B. V. Shabat, Hydrodynamic Problems and their Mathematical Models (Nauka, Moscow, 1973) (in Russian).

${ }^{17}$ B. W. Zeff, B. Kleber, J. Fineberg, and D. P. Lathrop, "Singularity dynamics in curvature collapse and jet eruption on a fluid surface," Nature (London) 403, 401 (2000).

${ }^{18}$ A. I. Fedorchenko and A. A. Chernov, "Formation of fingers at the front of an axially symmetric film of liquid upon the impact of a drop with a solid surface,” Dokl. Phys. 44, 570 (1999).

${ }^{19}$ N. E. Kochin, I. A. Kibel, and N. V. Roze, Theoretical Hydromechanics, edited by J. R. M. Radok, 5th ed. (Interscience, New York, 1964) (in Russian)

${ }^{20}$ A. I. Fedorchenko, "Melt drop spreading at collision with rigid surface," Schwerpunktprogram der Deutschen Forschungsgemeinschaft Anwendungsbezogene Optimierung und Steuerung, Augsburg, No. 361, 1992.

${ }^{21}$ J. Fukai, Z. Zhao, D. Poulikakos, C. M. Megaridis, and O. Miyatake, "Modeling of the deformation of a liquid droplet impinging upon a flat surface," Phys. Fluids A 5, 2588 (1993).

${ }^{22}$ P. A. Elmore, G. L. Chahine, and H. N. Oguz, "Cavity and flow measurements of reproducible bubble entrainment following drop impacts," Exp. Fluids 31, 664 (2001)

${ }^{23}$ A. A. Korobkin and V. V. Pukhnachov, "Initial stage of water impact," Annu. Rev. Fluid Mech. 20, 159 (1988).

${ }^{24}$ H. Lamb, Hydrodynamics (Cambridge University Press, Cambridge, England, 1962).

${ }^{25}$ I. M. Kirko, E. P. Dobychin, and V. I. Popov, "The phenomenon of the capillary bounce a ball at state of zero gravity," Dokl. USSR Acad. Sci. 192, 301 (1970).

${ }^{26} \mathrm{~S}$. T. Thoroddsen and K. Takehara, "The coalescence cascade of a drop," Phys. Fluids 12, 1265 (2000).

${ }^{27}$ S. Schiaffino and A. A. Sonin, "Molten droplet deposition and solidification at low Weber numbers," Phys. Fluids 9, 3172 (1997).

${ }^{28}$ J. Fink, D. Gault, and R. Greeley, "The effect of viscosity on impact cratering and possible application to the icy satellites of Saturn and Jupiter," J. Geophys. Res. B 89, 417 (1984). 